
Journal of Controlled Release. The final authenticated version is available online at: $\mathrm{https}: / /$ doi.org/10.1016/j.jconrel.2020.01.033

\title{
Evaluating single-domain antibodies as carriers for targeted vaccine delivery to the small intestinal epithelium
}

Shruti Bakshi ${ }^{\mathrm{a}, \mathrm{b}, \S}$, Raquel Sanz Garcia ${ }^{\mathrm{c}, \S}$, Hans Van der Weken ${ }^{\mathrm{c}}$, Ashuwini Tharad ${ }^{\mathrm{a}, \mathrm{b}}$, Shubham

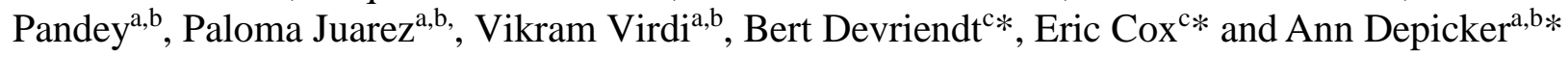

${ }^{a}$ Department of Plant Biotechnology and Bioinformatics, Ghent University, 9052 Gent, Belgium

${ }^{\boldsymbol{b}}$ VIB Center for Plant Systems Biology, 9052 Gent, Belgium.

${ }^{c}$ Laboratory of Immunology, Faculty of Veterinary Medicine, Ghent University, 9820 Merelbeke, Belgium.

§shared first authorship

*shared senior authorship, corresponding author

Ann Depicker: Anna.Depicker@UGent.be

Address- Department of Plant Biotechnology and Bioinformatics, Ghent University, Technologiepark 71, 9052 Ghent, Belgium

Eric Cox: Eric.Cox@UGent.be

Address- Department of Immunology, Salisburylaan 133, 9820 Merelbeke, Belgium.

Bert Devriendt: B.Devriendt@UGent.be

Address- Department of Immunology, Salisburylaan 133, 9820 Merelbeke, Belgium.

\begin{abstract}
Targeting a vaccine to the mucosal surface has recently been recognized as a promising approach to efficiently induce mucosal immune responses against enteric pathogens. However, poor uptake and inefficient transport of orally delivered subunit vaccines across the intestinal epithelium combined with weak immune responses still present important bottlenecks for mucosal vaccination. A possible strategy suggested to surmount these hurdles is to target the selected antigen to transcytotic receptors, such as aminopeptidase N (APN) present on enterocytes and antigen-presenting cells (APCs). Therefore, we aimed to identify potent and selective VHHs against porcine aminopeptidase $\mathrm{N}(\mathrm{pAPN})$, that were fused to the fragment crystallizable $(\mathrm{Fc})$ domain of the murine IgG2a, resulting in dimeric VHH-MG fusions. Out of a library of $30 \mathrm{VHH}-$
\end{abstract}


MG fusion candidates, two fusions displaying the best binding on pAPN-expressing cells were selected and showed in vivo internalization across the porcine gut epithelium. One of these fusions triggered systemic and intestinal IgA responses upon oral administration. Our results demonstrate the potential of bivalent VHH-MG fusions as delivery vehicles for vaccine antigens. VHHmediated and APN-targeted antigens to generate protective immunity at the mucosal surface remains to be further validated.

\section{Keywords:}

Antibody engineering, VHH-Fc, VHH, nanobodies, single-domain antibodies, protein expression, production, recombinant antibody, nanobody characterization, library screening, BioXP, Gibson cloning, vaccine targeting, intestinal infection.

\section{Abbreviations:}

pAPN, porcine aminopeptidase N; APC, antigen presenting cells; Fc, fragment crystallizable; MG, Fc domain of murine IgG; VHH, variable domain of heavy chain-only antibody; GBP, GFPspecific VHH; GI, gastrointestinal; mAb, monoclonal antibody; scFv, single-chain variable fragment; SEC, size exclusion chromatography.

\section{Introduction}

The vast majority of pathogens and external noxious substances enter the body through the mucosal surfaces of the respiratory, gastrointestinal (GI) and urogenital tracts, and thus mucosal immunity is of paramount importance to counter infection. Currently, needle-free oral vaccine delivery is the most attractive route of administration for protection against intestinal pathogens, because it offers many advantages, such as ease of administration, no risk of blood-borne infection and practicality for mass vaccination $[1,2]$. In contrast to subunit vaccines, only live attenuated vaccines or inactivated pathogen particles have been found to be effective in stimulating an efficient immune response. However, because of the safety issues associated with orally delivered 
live attenuated or inactivated pathogens, only a handful of such vaccines have been licensed. On the other hand, subunit vaccines are considered to be safe because they do not contain the live component of the pathogen, but poor transport of vaccine antigens across the intestinal epithelium to reach the underlying immune inductive sites resulting in a poor immune response pose a major hurdle to the development of oral vaccine delivery. Enterocytes or absorptive villus epithelial cells constitute $\sim 90 \%$ of all intestinal epithelium and they possess phagocytosis and transcytosis capacities to transport enteric pathogens or macromolecules across the epithelial barrier [3]. Therefore, antigen targeting to transcytotic receptors on enterocytes is an interesting approach for vaccine delivery and inducing a strong mucosal as well as systemic immune response against intestinal pathogens [4].

In the recent past, aminopeptidase $\mathrm{N}(\mathrm{APN})$ has been identified as a receptor for F4 fimbriae that is expressed on a variety of cells including small intestinal enterocytes and APCs, but not on epithelial cells of other parts of the GI tract [5]. Oral immunization with APN-specific polyclonal antibodies triggered mucosal immunity [5]. Moreover, functionalization of microparticles with APN-specific mouse monoclonal antibodies also increased uptake of these particles by the intestinal epithelium and triggered systemic immune responses [6]. These findings suggest that an APN-specific delivery agent conjugated with a vaccine antigen could be an interesting strategy to elicit a stronger intestinal immune response. To date, several targeting mechanisms have been identified, such as antibodies, cell-mediated targeting (DC immunotherapy, $\mathrm{T}$ cell adoptive transfer), and chemical conjugation of the antigen to small molecules such as glycans and amino acids [7]. Because of their strong affinity and specificity, antibodies are the ideal proteins to which an antigen of interest can be genetically fused for targeting to the APN-expressing enterocytes. Different antibody formats can be used, such as a monoclonal antibody (mAb), the single-chain variable fragments (scFvs), or the variable domain of heavy-chain-only antibodies (VHH, also known as nanobody). Although conventional mAbs have originally been preferred as a targeting vehicle, their production as fusion with the antigens turned out to be cumbersome and time-consuming. In addition, their structural complexity hampers their use as potential ligands. Therefore, other simpler forms such as scFvs and VHHs were considered as alternative approaches to full-sized antibodies. Although the advances in antibody engineering made it possible to easily design and clone $\mathrm{scFv}$ genes based on the targeting performance of mAbs, the stability and accumulation of the synthetic scFv proteins vary heavily, 
depending on several factors, including the unpredictable length of the requisite linker to obtain proper folding and the assembly of the two variable domains, and this often restricted $\mathrm{scFv}$ targeting applications $[8,9]$. On the other hand, the VHH immunoglobulin domain is only a $15-\mathrm{kDa}$ variable fragment derived from the camelid heavy-chain only antibody [10]. Unlike scFv, VHH contains its antigen-binding properties in a single domain rather than in two variable domains in conventional antibodies and derived scFvs, while retaining similar affinity. Owing to its peculiar properties such as small size, ease of production, good thermostability and efficient tissue penetration, $\mathrm{VHH}$ has received great interest for various applications compared with classical antibodies [11-13].

The present study focuses on evaluating the VHH domain as a carrier for targeted vaccine delivery to the gut epithelium to induce strong mucosal immune responses. We identified a panel of $28 \mathrm{VHHs}$ by immunizing llama with porcine APN (pAPN) and screening on pAPN-expressing cell lines. The selected binders belong to at least 14 different families based on their similarity (>80\% sequence identity) in the CDR3 sequence. In order to increase affinity and avidity and at the same time allow easy purification, these $\mathrm{VHH}$ domains were fused to the Fc domain of murine IgG2a, hereafter, referred to as VHH-MG fusions.

Nowadays, different production platforms such as mammalian cells, yeast, plants, etc. are used for the production of complex assembled glycoproteins such as antibodies. Production based on mammalian cells, like Chinese Hamster Ovary (CHO) cells is straightforward for the production of mAbs but is complicated, expensive and often associated with the risk of animalderived products when synthetic fusions need to be produced [14-16]. Therefore, transgenic plants and yeast expression systems show a great potential. Yeast such as Pichia pastoris possesses a clear advantage over plant expression systems in terms of short generation time, cost effectiveness, ease of genetic manipulation and scalability [17, 18]. Moreover, P. pastoris secretes very low amount of endogenous proteins, therefore secreted recombinant proteins are highly enriched, which facilitates downstream processing $[19,20]$. On the other hand, the glycosylation profile of recombinant proteins in plants is much more alike that of animal cells than the yeast expression platform (see ref. [21]).

Here, we chose the Pichia platform as a more convenient, faster and flexible platform to test the production of different pAPN-binding VHH-MG fusion proteins and their capacity to raise mucosal immune responses. The yeast-produced VHH-MG fusions were further characterized for 
their binding to and uptake in pAPN-expressing cells. At least two VHH-MGs were produced to high levels, appeared to be stable and were efficiently endocytosed by cell lines and in loops of the porcine gut under physiological conditions. One of these fusions elicited systemic and intestinal IgA responses.

\section{Materials and methods}

\subsection{Vectors, strains, and cell lines}

For the VHH library preparation, the pMECS phage display vector system was used that contains the pelB signal sequence to secrete the expressed VHH in bacterial periplasm and that adds an HA and $\mathrm{His}_{6}$ tag at the $\mathrm{VHH}$ carboxy-terminal end, enabling purification. The yeast expression vector pPICZalphaH6E (NCBI accession number KM035419.1) modified for GoldenBraid cloning (named pKaiGG) was used for cloning the VHH-MG fusions under the control of the methanolinducible $A O X 1$ promoter. The vector contains the Zeocin resistance marker for selection in bacterial as well as yeast cells. E. coli strains TG1 and DH5 $\alpha$ were used for construction of the VHH library and fusion expression vectors, respectively. The VHH-MG fusions were expressed in the P. pastoris wild-type strain NRRL Y-11430. Stable APN-expressing cell lines (intestinal porcine epithelial cells IPEC-J2 and baby hamster kidney BHK-21) were obtained by transfection and cultured as previously described $[5,6]$.

\subsection{Purification of intestinal APN}

Enterocytes were isolated from the small intestine of a 3-week-old piglet following the method of Lundqvist, Hammarström, Athlin and Hammarström [22]. After analysis using light microscopy, more than $85 \%$ enterocyte purity was observed. Next, brush border membrane vesicles (BBMVs) were isolated from the enterocytes as described by Melkebeek, Rasschaert, Bellot, Tilleman, Favoreel, Deforce, De Geest, Goddeeris and Cox [5]. The obtained BBMVs were subsequently lysed in 9 volumes of lysis buffer $(100 \mathrm{mM}$ Tris-HCl, $300 \mathrm{mM} \mathrm{NaCl}, 1 \%$ Triton X-100, 0.5\% sodium deoxycholate, 0.1\% SDS, $2 \mathrm{mM}$ leupeptin, $5 \mathrm{mM}$ DTT, $1 \mathrm{mM}$ AEBSF), sonicated for 2 min on ice, rotated for $30 \mathrm{~min}$ at $4^{\circ} \mathrm{C}$ and centrifuged at $17,400 \mathrm{~g}$ for $15 \mathrm{~min}$ at $4^{\circ} \mathrm{C}$. Supernatant was collected and dialyzed in PBS using a 10-kDa MWCO dialysis cassette (Thermo scientific). 
Protein concentration was determined by BCA reaction (Pierce BCA protein assay kit), following the manufacturer's recommendations. Lysates were stored at $-20^{\circ} \mathrm{C}$ until further use.

Intestinal pAPN was purified by immunoprecipitation from these lysates. First, affinitypurified rabbit anti-APN IgG (in house) was crosslinked to protein A Sepharose beads (CL-4B, Sigma). For this, $50 \mathrm{mg}$ of beads were washed three times in $1 \mathrm{ml}$ distilled water to remove salts by centrifugation at $400 \mathrm{~g}$ for $2 \mathrm{~min}$. Rabbit anti-APN $\operatorname{IgG}(4 \mathrm{mg})$ was added to the beads for $1 \mathrm{~h}$ in PBS at room temperature (RT). Next, beads were washed twice with $1 \mathrm{ml} 0.2 \mathrm{M}$ sodium borate buffer ( $\mathrm{pH} 8.0)$, after which $1 \mathrm{ml} 0.2 \mathrm{M}$ sodium borate $(\mathrm{pH} 9.0)+20 \mathrm{mM}$ dimethylpimelimidate (DMP, Sigma) was added for $30 \mathrm{~min}$ at RT. After crosslinking, the beads were washed twice for $1 \mathrm{~h}$ in $1 \mathrm{ml} 0.2 \mathrm{M}$ ethanolamine $+50 \mathrm{mM}$ ammonium bicarbonate buffer ( $\mathrm{pH} \mathrm{8.0)}$. Next, the beads were washed thrice in PBS before elution buffer (0.2 M Glycine-HCl, $50 \mathrm{mM}$ L-arginine, $1 \mathrm{M}$ $\mathrm{NaCl}$ ( $\mathrm{pH}$ 2.0)) was added to remove non-crosslinked IgGs. Beads were washed again three times in PBS and stored at $4^{\circ} \mathrm{C}$ until further use. Finally, the anti-APN-beads were incubated with $1 \mathrm{ml}$ of BBMV lysate overnight at $4^{\circ} \mathrm{C}$ on a rotating wheel. Beads were centrifuged $\left(400 \mathrm{~g}, 2 \mathrm{~min}, 4^{\circ} \mathrm{C}\right)$ and supernatant was collected. Beads were washed thrice with PBS to remove unbound proteins. Next, APN was eluted from the beads with elution buffer for $10 \mathrm{~min}$ at RT. After centrifugation, supernatant was collected and neutralized with $1 \mathrm{M}$ Tris- $\mathrm{HCl}(\mathrm{pH} 9.0)$. Eluted APN was dialyzed immediately in PBS and stored at $-20^{\circ} \mathrm{C}$. Concentration was determined with the Pierce BCA protein assay kit and purity ( $>95 \%$ ) was assessed by performing a silver staining (Pierce® silver stain kit; Thermo Scientific), following the manufacturer's instructions.

\subsection{Generation of a pAPN-specific VHH library}

Porcine APN-specific VHHs were developed by the VIB Nanobody Core facility, Brussels (Belgium), as described previously [23]. Briefly, two llamas were immunized subcutaneously on days $0,7,14,21$ and 28, each time with $320 \mu \mathrm{g}$ (per animal) APN isolated from porcine kidney (Sigma, cat. No. L6007-50UN). On day 35, each llama received a final booster with $200 \mu \mathrm{g}$ of APN isolated from pig intestine. The porcine intestinal APN was purified as described above. For all immunizations, Gerbu P (GERBU Biotechnik) was used as adjuvant. On day 40, anticoagulated blood was collected for lymphocyte preparation. Total RNA from peripheral blood lymphocytes (PBLs) was used as template for first strand cDNA synthesis with an oligo(dT) primer. Using this cDNA, the VHH-encoding sequences were amplified by PCR, digested with PstI and NotI, and 
cloned into the PstI and NotI sites of the phagemid vector pMECS, and in this way fused to a histidine tag. Electrocompetent E. coli TG1 cells were transformed with the recombinant pMECS vector. An independent VHH library was obtained from each of the llamas, and each was subjected to a panning (in solution), performed on stably transfected BHK-21 cells expressing pAPN. The phage outputs of the first panning round from the two libraries were then pooled and the pool was used for further panning rounds. 190 randomly selected colonies from the second and third panning rounds (95 from each round) were sequenced and the 28 obtained unique VHH sequences were aligned and grouped in families according to the similarity in their CDR3 sequences as shown in Supplementary Fig. S1. Using crude periplasmic extracts, 28 unique VHH (Nanobody) sequences were analyzed by flow cytometry for specificity to pAPN using pAPN-expressing BHK-21 cells.

\subsection{Construction of the $\mathrm{VHH}-\mathrm{MG}$ fusion expression vectors}

To clone the in silico designed VHH-MG fusions in the yeast expression vector pKaiGG, customized reagents designed for Gibson Assembly® cloning were obtained from SGI-DNA (La Jolla, California), being synthetically produced DNA fragments of the VHH-encoding sequences fused to the Fc tail of mouse IgG2a (Hinge-CH2-CH3; NCBI accession number KC295246.1) with 40-base overlaps between the insert and the vector. The expression vector pKaiGG adapted for Golden Gate cloning was digested with SapI and column purified (GeneJET PCR purification kit, ThermoFisher Cat. No. K0701). The VHH fragments were always assembled with the same Fc fragment and were cloned at the 5 'end in frame with the alpha factor secretion signal sequence (from novel $P$. pastoris expression vector named pPICZH6E; GenBank accession number KM035419) and at the 3 'end with the transcription termination sequence into the in-house SapIdigested pKaiGG vector via the BioXp ${ }^{\mathrm{TM}} 3200$ System in an approximately 18-h reaction and transformed into E. coli DH5 $\alpha$. Upon plasmid preparation and sequence confirmation of the insert, yeast cells were transformed with the PmeI-linearized vectors and positive transformants were selected on YPD (1\% yeast extract, $2 \%$ peptone, $2 \%$ Dextrose) supplemented with $50 \mathrm{mg} / \mathrm{ml}$ Zeocin (Invitrogen).

\subsection{Production of VHH-MG fusions in P. pastoris}


Expression and secretion of the VHH-MG fusions in the medium was first analyzed in 2-ml cultures. On day one, $2 \mathrm{ml}$ of BMGY medium (1\% Bacto yeast extract, $2 \%$ peptone, $1.34 \% \mathrm{YNB}$, $0.1 \mathrm{M}$ potassium phosphate (pH 6), $1 \%$ glycerol) with $25 \mathrm{mg} / \mathrm{ml}$ Zeocin was inoculated with individual transformants and incubated while shaking at $28^{\circ} \mathrm{C}$ for $48 \mathrm{~h}$. For each VHH-MG construct, four independent transformants were grown in 24-well plates. The cells were then pelleted for $10 \mathrm{~min}$ at $1500 \mathrm{~g}$ and resuspended in $2 \mathrm{ml} \mathrm{BMMY}$ medium (1\% Bacto yeast extract, $2 \%$ peptone, $1.34 \%$ YNB, $0.1 \mathrm{M}$ potassium phosphate (pH 6), $1 \%$ methanol) to induce expression of VHH-MG. Cultures were incubated while shaking at $28^{\circ} \mathrm{C}$ for $48 \mathrm{~h}$ and spiked with $1 \%$ methanol (v/v) every $12 \mathrm{~h}$. After $48 \mathrm{~h}$ of induction, the yeast cells were pelleted, and the supernatant was analyzed by SDS-PAGE, western blot and ELISA to assess the accumulation levels of the secreted VHH-MG fusions in the medium.

\subsection{Affinity purification of $\mathrm{VHH}-\mathrm{MG}$ fusions}

Yeast transformants that yielded high levels of VHH-MG were selected for upscaling in 1-1 cultures, using similar growth and induction conditions as described above for 2-ml cultures. $P$. pastoris cultures grown in baffled flasks were harvested after $48 \mathrm{~h}$ of induction. Culture supernatant was filtered using $0.22-\mu \mathrm{M}$ PES membrane filters (Millipore) and affinity purified on a 1-ml HiTrap MabSelect SuRe protein A column (GE healthcare), equilibrated with $20 \mathrm{mM}$ sodium phosphate, $300 \mathrm{mM} \mathrm{NaCl}$ buffer, $\mathrm{pH}$ 7.8. Bound protein was eluted with $1 \mathrm{M}$ arginine, $\mathrm{pH}$ 2.7 and immediately neutralized with $1 \mathrm{M}$ Tris, $\mathrm{pH}$ 9. Relevant fractions with high concentration of VHH-MG were pooled and subjected to size-exclusion chromatography (Superdex 200, GE Healthcare). Fractions containing the major antibody peak in the chromatogram were pooled, concentrated by dialyzing against $20 \%$ polyethylene glycol in PBS and stored at $-80^{\circ} \mathrm{C}$ until use. The protein concentration was determined by $\mathrm{OD}_{280}$ measurement.

\subsection{SDS-PAGE and western blotting}

VHH-MG fusions (crude/purified) were analyzed on a commercial 4 to $20 \%$ gradient gel (Bio$\mathrm{Rad}$ ) under reducing conditions and stained with Coomassie G-250 following the manufacturer's instructions. For western blotting, proteins were transferred onto a polyvinylidene difluoride membrane (PVDF) using the semidry transfer method (Bio-Rad). Blotted membrane was blocked overnight with $2 \%$ skimmed milk in PBST (PBS $+0.1 \%(\mathrm{v} / \mathrm{v})$ Tween-20) at $4^{\circ} \mathrm{C}$. Thereafter, the 
membrane was probed with anti-mouse IgG (GE Healthcare, NXA931) conjugated to horseradish peroxidase (HRP), diluted 1:2000 in blocking solution and incubated for $1 \mathrm{~h}$ at RT. The membrane was then washed three times with PBST, and bands were visualized by adding HRP substrate (WesternBright ECL, Advansta). The membrane was imaged using a ChemiDoc MP imaging system (Bio-Rad) and analyzed by ImageLab software (Bio-Rad).

\subsection{Antigen-binding ELISA}

96-well ELISA plates (Nunc Polysorp®) were coated for two h at $37^{\circ} \mathrm{C}$ with $400 \mathrm{ng}$ of APN (Sigma) diluted in PBS. Plates were then washed three times with PBST and blocked overnight at $4^{\circ} \mathrm{C}$ with $3 \%(\mathrm{w} / \mathrm{v})$ gelatin prepared in PBS and $0.05 \%$ Tween-80. Following three washes with PBST, VHH-MG fusions were serially diluted in dilution buffer $(2 \%$ skimmed milk + PBS + $0.05 \%$ Tween-20), added to each well and incubated for $1 \mathrm{~h}$ at RT. An in-house produced pAPNspecific mAb (IMM013, see ref. [6]) was used as a positive control, while a GFP-specific VHH fused to a murine IgG3 (GBP-MG) was used as a negative control [24]. After washing, bound VHH-MG fusions were detected by anti-mouse IgG (GE Healthcare, NXA931), diluted 1:5000 in the dilution buffer. Following incubation for $1 \mathrm{~h}$ at RT, plates were washed three times with PBST and developed by adding HRP substrate (one SIGMAFAST ${ }^{\mathrm{TM}}$ OPD tablet dissolved in $20 \mathrm{ml}$ deionized water). Finally, the reaction was stopped with $1 \mathrm{M}$ hydrochloric acid and the optical density of the colorimetric reaction was measured at $492 \mathrm{~nm}\left(\operatorname{VersaMax}^{\mathrm{TM}}\right)$.

\subsection{Flow cytometry}

Binding of the different VHH-MG fusions to APN-expressing cell lines was analyzed using flow cytometry. The wild-type and APN-transfected IPEC-J2 and BHK-21 cell lines were grown until reaching a 90\% confluence and detached with StemPro accutase (Gibco). Detached cells (3.0 x $10^{5}$ ) were transferred to a conical bottom 96-well microtiter plate (Gibco) in 200- $\mu 1$ culture medium and centrifuged for $3 \mathrm{~min}$ at $350 \mathrm{~g}$ and $4^{\circ} \mathrm{C}$. Cells were incubated with $2 \mu \mathrm{g} / \mathrm{ml} \mathrm{VHH-MG}$ fusions or anti-APN mAb (clone IMM013) on ice for $30 \mathrm{~min}$. Detection of bound VHH-MG fusions was performed with an AF647-conjugated anti-mouse IgG2a antibody (4 $\mu \mathrm{g} / \mathrm{ml}$; Invitrogen, A21241) and with an AF647-conjugated anti-mouse IgG1 antibody (4 $\mu \mathrm{g} / \mathrm{ml}$; Invitrogen, A-21240) for IMM013. Cells were incubated for $30 \mathrm{~min}$ on ice. Dead cells were 
excluded using Sytox blue staining ( $5 \mathrm{nM}$; Molecular probes). A total of 10,000 viable, single cells was measured for each condition (Cytoflex, Beckman Coulter).

\subsection{VHH-MG endocytosis assay}

APN-expressing BHK-21 cells were seeded in 24 -well plates $\left(1.0 \times 10^{5}\right.$ cells in $\left.1 \mathrm{ml}\right)$ on top of a sterile cover slip and cultured until a monolayer was formed. Cells were washed twice with icecold PBS and stored on ice before the VHH-MG fusions or IMM013 were added to the cells (250 $\mu \mathrm{l} ; 100 \mu \mathrm{g} / \mathrm{ml})$ in ice-cold culture medium. After a 60 -min incubation at $4^{\circ} \mathrm{C}$, cells were washed thrice with ice-cold PBS and incubated for 30 min at $37^{\circ} \mathrm{C}, 5 \% \mathrm{CO}_{2}$ in warm culture medium. After washing twice in PBS, cells were fixed for 10 min with $500 \mu 14 \%$ paraformaldehyde. Next, the presence of VHH-MGs or IMM013 on the cell membrane was detected with an AF568conjugated anti-mouse IgG $(\mathrm{H}+\mathrm{L})(2 \mu \mathrm{g} / \mathrm{ml}$; Invitrogen, A-11004) for $30 \mathrm{~min}$ at RT and protected from light. After washing three times with PBS $+1 \%$ FCS, cells were permeabilized with $250 \mu 1$ $0.2 \%$ Triton-X100 for 2 min and washed again with PBS + 1\% FCS. Both VHH-MG and IMM013 were then detected using a FITC-conjugated anti-mouse IgG (whole molecule) (Sigma, F2883) for $30 \mathrm{~min}$ at RT, protected from light. After washing three times, the cover slip was taken out and mounted on a microscope slide in mounting solution (DABCO). Images were taken with a confocal microscope (Leica).

\subsection{In vivo uptake of anti-APN VHH-MG}

Three female, 5-week-old piglets were used to assess the uptake of the VHH-MGs in gut ligated loops as described previously [25]. Briefly, following anesthesia and laparotomy, the jejunum was localized and six 3-cm loops with 10-cm intervals between each loop were made avoiding Peyer's patches. Blood supply was assured by placing the ligatures between the mesenteric arcades. The pAPN-specific mAb (IMM013, see ref. [6]) was used as a positive control, while GBP-MG was used as a negative control [24]. The anti-APN mAb IMM013 (1 mg) or an equimolar amount of the different VHH-MGs were diluted in $3 \mathrm{ml}$ of PBS and injected in the lumen of the loops. Upon injection, each loop was returned to the abdominal cavity and the abdomen was closed. After a 5-h incubation, the animals were euthanized with an overdose of sodium pentobarbital and tissue samples from intestines, loops and mesenteric lymph nodes were collected. Tissue samples were embedded in $2 \%$ Methocel ${ }^{\circledR} \mathrm{MC}$ (Fluka), snap frozen in liquid nitrogen and stored at $-80^{\circ} \mathrm{C}$ until 
use. All animal procedures were approved by the Ethical Committee of the Faculty of Veterinary Medicine of Ghent University (EC 2018-04).

\subsection{Immunohistochemistry}

Cryosections $(8 \mu \mathrm{m})$ of porcine ileum obtained from 3-week-old piglets or from the gut loops were cut with a Leica CM3050 S cryostat, mounted on APES-coated glass slides, dried $\left(30 \mathrm{~min}, 40^{\circ} \mathrm{C}\right)$ and fixed in acetone during $10 \mathrm{~min}$ at $-20^{\circ} \mathrm{C}$. Subsequently, the cryosections were incubated during $30 \mathrm{~min}$ in ammonium chloride buffer $(50 \mathrm{mM}, \mathrm{pH} 8)$ and washed thoroughly. All washing steps were carried out at RT in PBS. Fc receptors were blocked for 30 min at $37^{\circ} \mathrm{C}$ with $\mathrm{PBS}$ containing $10 \%$ sheep or goat serum. Binding of the VHH-MG or IMM013 was detected by incubation for $1 \mathrm{~h}$ at $37^{\circ} \mathrm{C}$ with a FITC-conjugated sheep anti-mouse IgG $(10 \mu \mathrm{g} / \mathrm{ml}$; Sigma; F2883) or goat antimouse IgG2a. Nuclei were counterstained with Hoechst $(10 \mu \mathrm{g} / \mathrm{ml})$. To assess endocytosis sections were stained with a rabbit anti-pan-cytokeratin antibody (Abcam, ab9377) and detected with a TexasRed-conjugated anti-rabbit IgG. The sections were washed in ultrapure water and mounted in mounting solution (DABCO). Tissues were imaged with a Leica DC 100 fluorescence microscope mounted with a Scion Corporation camera or a Leica confocal microscope. Images were analyzed and processed using Fiji.

\subsection{Oral immunization experiment}

All animal procedures were approved by the ethical committee of the Faculty of Veterinary Medicine (EC 2018-51). Twelve conventionally reared piglets (Belgian Landrace x Pietrain) from a Belgian farm were weaned at 3 weeks of age and transported to our facilities. Animals were treated with colistin sulphate (Promycine ${ }^{\circledR}, 100.000$ U.I./kg of animal weigh) for 5 days before the start of the experiment. The design of the oral immunization experiment is depicted in Fig. 7a. Animals were randomly divided in three groups: a mouse IgG2a control group and two groups receiving either the APN-specific VHH-MGs 2L65 or 3L94. Animals were orally immunized on three consecutive days followed by a booster immunization on day 14 post primary immunization. The $\mathrm{HCl}$ production in the stomach was blocked by administering Omeprazole (20 mg/animal) 24 hours before each immunization and animals were deprived of feed 12 hours before the immunizations. The animals were orally immunized with either $1 \mathrm{mg}$ of 3L94-MG, 2L65-MG or an equimolar amount of an irrelevant mouse IgG2a (Bio X cell; West Lebanon, USA), adjuvanted 
with $50 \mu \mathrm{g}$ of cholera toxin (Merck, ref: C8052). Blood was collected on days 0, 9, 14, 21 and 28 post primary immunization (ppi) to analyze the elicited immune responses. At day 28 ppi animals were euthanized by intravenous injection of sodium pentobarbital $20 \%$ (60 mg/2.5 kg; Kela) and upon exsanguination small intestinal tissue samples were collected.

\subsection{Mouse IgG2a ELISA}

Blood was taken from the jugular vein into a gel and clot activator tubes (Vacutest, Kima). After $1 \mathrm{~h}$ incubation at room temperature, tubes were centrifuged, and serum was collected and inactivated at $56^{\circ} \mathrm{C}$ during $30 \mathrm{~min}$. Serum samples were stored at $-20^{\circ} \mathrm{C}$ until use.

An in-house produced mouse IgG2a monoclonal antibody was coated in 96-well microtiter plates (Polysorp; Life Technologies) at $6 \mu \mathrm{g} / \mathrm{ml}$ in PBS for $2 \mathrm{~h}$ at $37^{\circ} \mathrm{C}$. Upon overnight blocking at $4^{\circ} \mathrm{C}$ in PBS supplemented with $0.2 \%$ Tween 80 and $3 \%$ BSA, diluted serum samples (start dilution 1:10 in dilution buffer) were added to the wells. Upon incubation for $1 \mathrm{~h}$ at $37^{\circ} \mathrm{C}$, plates were washed and incubated for $1 \mathrm{~h}$ at $37^{\circ} \mathrm{C}$ with HRP-conjugated mouse anti-swine IgG (MT424, 1/1000; MabTech, Nacka Strand, Sweden) or HRP-conjugated goat anti-swine IgA (1/10.000; Bethyl; Montgomery, Texas, U.S). Following three washing steps, 2,2'-azino-bis(3ethylbenzothiazoline-6-sulphonic acid) (ABTS) substrate was added and the optical density was measured at $405 \mathrm{~nm}$ after $45 \mathrm{~min}$ incubation at $37^{\circ} \mathrm{C}$ using a spectrophotometer (Tecan SpectraFluor).

\subsection{ELIspot}

Blood was taken on heparin from the jugular vein of piglets and PBMCs were isolated by density gradient centrifugation using Lymphoprep® (Alere Technologies, Oslo, Norway). Erythrocytes were lysed in ammonium chloride solution. The resulting PBMC fraction was washed twice in ice cold PBS + 1 mM EDTA, resuspended in CTL-test BTM (Cellular Technology Limited, Cleveland, USA) supplemented with $1 \%$ penicillin $(100 \mathrm{IU} / \mathrm{mL})$, streptomycin $(100 \mu \mathrm{g} / \mathrm{mL})$ and kanamycin to 1 x107 cells/ml. Mononuclear cells (MCs) were isolated from mesenteric lymph nodes (MLN), jejunal Peyer's Patches (JPP), jejunal lamina propria (JLP), ileal Peyer's Patches (IPP) and ileal lamina propria (ILP) and processed as described previously [26]. Isolated MC were resuspended in leukocyte medium (RPMI-1640 (Gibco) containing 10\% fetal calf serum, 1mM 
sodium pyruvate, $2 \mathrm{mM}$ l-glutamine, penicillin (100 IU/mL), streptomycin $(100 \mu \mathrm{g} / \mathrm{mL})$, and nonessential amino acids (1\%) and counted.

MultiScreen filter plates (96-well format, MAIPA4510, Millipore) were activated with $70 \%$ ethanol, washed with ultrapure (UP) water and coated with $10 \mu \mathrm{g} / \mathrm{ml}$ mouse IgG2a overnight at $4^{\circ} \mathrm{C}$. Upon washing, the plates were incubated for $2 \mathrm{~h}$ at $37^{\circ} \mathrm{C}$ with CTL-test $\mathrm{B}$ medium. Mononuclear cells (2.5x105/well) from each tissue were added to the wells and incubated for $18 \mathrm{~h}$ at $37^{\circ} \mathrm{C}$ in a humidified $5 \% \mathrm{CO} 2$ atmosphere. Cells were then removed by intensive washing with PBS containing 0.1\% Tween20. Upon washing, HRP-conjugated anti-swine IgG (MT424, 1/1000; MabTech) or IgA (1/10.000; Bethyl laboratories) was added in assay buffer (PBS containing $0.1 \%$ Tween 20 and $0.1 \%$ BSA) and incubated for 1 hour at room temperature. Finally, 3,3',5,5'Tetramethylbenzidine (TMB) substrate for membranes (Sigma) was added to the wells after 3 washing steps. The reaction was stopped by intensive washing with UP water and the plates were allowed to dry overnight at $4^{\circ} \mathrm{C}$. Images were taken using an immunospot reader (Luminoskan). Spots were counted manually based on size discrimination.

\subsection{Statistical analysis}

The data were analyzed using GraphPad Prism 6. Serum antibody levels and the number of circulating antigen-specific antibody secreting cells were analyzed with two-way ANOVA and Dunnett's test for multiple comparisons. The number of intestinal antigen-specific antibody secreting cells were analyzed with a t-test and Holm-Sidak for multiple comparisons. The significance level was set at 0.05 .

\section{Results}

\subsection{Construction of an APN-specific VHH library}

To prepare an anti-APN VHH library, two llamas were immunized five times with commercially available kidney pAPN. However, because we wanted to target intestinal pAPN and not kidney pAPN, which are encoded by the same gene but might differ in glycosylation pattern and hence in the accessibility of particular antibody epitopes, the sixth booster was given with intestinal pAPN prepared by a lengthy procedure as described in the Materials and Methods section. Two independent VHH-phage display libraries were constructed, each consisting of about $10^{8}$ 
transformants. After two consecutive rounds of panning, performed on stably transfected BHK21 cells expressing pig aminopeptidase N, 190 clones were randomly selected and sequenced. Through comparative alignment, VHHs having more than $80 \%$ percent sequence homology in their CDR3 region (B-cell lineages) were grouped as one family because they most likely recognize the same epitope (see ref. [27]) but their characteristics (e.g. affinity, expression yield, stability etc.) can be different. In this way, we obtained 28 unique VHHs belonging to 14 different families as candidate binders to APN (Supplementary Fig. S1). These 28 unique VHHs were produced in E. coli cells and the crude periplasmic extracts were analyzed for their binding specificity via flow cytometry on pAPN-expressing BHK21 cells. The parental non-transfected BHK-21 cells served as negative control cells. An irrelevant VHH, (BCII10 specific for bacterial $\beta$-lactamase, described by Conrath et al.[28]) and a mouse anti-APN mAb IMM013 [6] were used as negative and positive control, respectively. This differential FACS analysis confirmed 22 of the originally 28 screened different VHHs belonging to 11 different families specific for porcine APN, demonstrating that the panning of the library on pAPN-transfected cells highly enriched for APNbinding VHH clones (Supplementary Fig. S2).

\subsection{Construction of a VHH-MG expression vector via the BioXP DNA printer}

Because the FACS analysis for the panning on APN-transfected BHK-21 cells was performed with crude periplasmic extracts, the quality of binding of the different VHHs had to be further analyzed with purified proteins to allow comparison among the different VHHs and to rule out any experimental variables such as nanobody expression, periplasmic extraction efficiency, etc in the FACS analysis (Supplementary Fig. S2). Important to realize is that some of the VHHs may contain an amber stop codon within the VHH sequence, because the VHH library with C-terminal histidine tag was prepared in the suppressor E. coli strain TG1 that reads the amber stop codon (TAG) as glutamine (Q). Thus, when an amber codon was identified by sequencing, it was also replaced by the glutamine codon.

Classically the selected VHHs are sub-cloned in direct fusion with an affinity tag in a nonsuppressor E. coli strain, but here we followed a new strategy. We sub-cloned the VHHs in direct fusion with an Fc fragment to have the fusions produced in the medium of Pichia pastoris, providing the following advantages: Fc can be used as an affinity tag for protein purification, it makes the VHH-Fc fusion bivalent providing an avidity effect, and it allows comparison with the 
mouse monoclonal antibody IMM013 for APN binding as positive control. Twenty-eight selected VHHs were fused to the Fc domain of mouse IgG2a and cloned into an in-house P. pastoris expression vector. Two irrelevant VHHs, V2 [29] and D3 [30], specific for the F4 and F18 fimbriae of enterotoxigenic $E$. coli, respectively, were included as negative control.

The sequences of the VHH and Fc domain were synthesized using the BioXPTM 3200 System and transformed into the yeast expression vector (Fig. 1a). All 30 constructs (with 28 selected VHHs and 2 negative controls) successfully built with the BioXP DNA printer were transformed into E. coli and four colonies per construct were analyzed by colony PCR or restriction digestion followed by sequencing to identify clones with the right insert. Twenty-three out of 30 constructs $(77 \%)$ resulted in an error-free clone by sequencing only one colony, while for seven other VHH-MG constructs, two colonies had to be analyzed to obtain the right clone; for only one clone, i.e. $2 \mathrm{~L} 58 \mathrm{MG}(3 \%)$ the sequence was still incorrect in all four colonies, having one mutation in the coding sequence (Fig. 1b). Coincidently, this incorrect clone from family 12 was also negative in panning during VHH library preparation (Supplementary Fig. S2). Therefore, we omitted it from the further analyses.

\subsection{Yeast-produced VHH-MG fusions bind efficiently to full-length, surface-expressed APN}

The 29 obtained VHH-MG encoding constructs were then transformed into Pichia, and for each, four individual yeast colonies were screened to identify the transformant producing the highest amount of VHH-MG accumulating in the culture medium. Therefore, equal amounts of supernatant were analyzed by SDS-PAGE and by western blotting to examine the intensity of the recombinant protein signal and the integrity of the protein (Supplementary Fig. S3). Only for the best expressing constructs, a band could be observed after SDS-PAGE; but for most constructs, a band was clearly present at the expected molecular weight after western blot analysis. The concentration of the different VHH-MGs produced in P. pastoris was determined using purified V2-MG and D3-MG reference proteins, and this ranged from 4 to $60 \mathrm{mg} / \mathrm{l}$ in 2-ml cultures, based on the analysis by ImageLab software. Only for some fusions, such as 3L11MG (family 10), 2L22MG (family 5) and V2-MG, a degradation product was detected besides the full-length product, suggesting that the VHH has an effect on the stability of the VHH-MG fusion as was also seen for VHH-IgA fusions [31]. 
Next, we determined the binding specificity of the VHH-MG fusions in a kidney APNspecific ELISA (Fig. 2a). Three-fold dilution series of VHH-MG in the culture supernatant were probed with anti-mouse IgG. The three strongest VHH-MGs, i.e. 2L48MG, 3L94MG and 2L76MG, displayed a strong binding activity to immobilized APN, each belonging to a different CDR3 group. These three VHH-MGs also showed good binding in the FACS assay performed after the panning to screen for phage displayed VHHs (Supplementary Fig. S2). Interestingly however, some families of VHH-MG fusions were completely negative in the ELISA, for instance family 4 (2L63-MG, 2L69-MG), while they were strong binders in the FACS assay (Supplementary Fig. S2). It is thus important to note that the relative binding activity on APNcoated wells in the ELISA assay did not correlate with the affinity of the VHH-MG fusions for the cell-expressed APN. Notably, VHH screening was performed via FACS using the cells expressing APN on their surface, so binding on immobilized APN might differ with binding on cells, perhaps because epitopes become less accessible during immobilization. Also, because the experiments were performed with crude medium samples, in which the VHH-MG protein accumulation was not equilibrated, the ELISA signal could be related to the protein concentration in the culture medium rather than to the affinity. (Fig. 2a, Supplementary Fig. S2).

VHH-MG fusions were further assessed for their binding specificity and affinity on parent cell lines versus the derived transfected APN-expressing cell lines (BHK21 and IPEC-J2) via flow cytometry with equal amounts of VHH-MG protein (Fig. 2b). Of note, IPEC-J2 are porcine small intestinal epithelial cell lines which express low levels of APN on their surface and hence, only stably transfected cells were used. Based on the relative VHH-MG concentration, as determined by western blotting, equal molar amounts of the VHH-MG proteins and the positive control (IMM013) were spiked into spent medium of P. pastoris. Several strong APN-binders were identified based on the number of cells with bound VHH-MG through flow cytometry, whereas no binding was observed of spent medium with non-transfected BHK21/IPEC-J2 cells. Interestingly, family 4 (2L63 and 2L69), which was negative in an APN-specific ELISA, turned out to contain strong binders in the cell-based binding assay (Fig. 2b, Supplementary Fig. S3). These findings were in line with the FACS data obtained from the crude periplasmic extract during VHH library screening (Supplementary Fig. S2). We found that the amplitude of the binding activity on IPEC-J2-APN cells in comparison to BHK21-APN was significantly lower, which may be related to the amount of APN expressed on the IPEC-J2 cell membrane. 
We selected at least on clone from the families showing binding activity in the flow cytometric screening (excluding family 6,10,11 and 14) to further assess their affinity for small intestinal tissue via immunohistochemistry (IHC). Based on their binding activity, some clones were selected and purified for the in vivo analysis. Clones with low yield such as 2L76-MG, 2L34MG and 2L52 were omitted from further analysis. Therefore, in Figure 3, only six families containing strong $(1,4,8,9)$ and moderate binders $(5,12)$ are shown that were selected for upscaling. As can be seen in Figure 3, each VHH-MG displayed binding to the intestinal ileum, whereas all controls were negative and did not show background; moreover, the intensity of the signal correlated with the binding activity in the FACS analysis (Fig. 2b).

\subsection{Purified APN-binding VHH-MG fusions are internalized by intestinal epithelial cells}

For each family, a single VHH-MG fusion displaying high expression and secretion in the spent Pichia medium and showing strong binding in FACS, was selected for upscaling. Secreted VHHMG fusions were affinity purified on protein $\mathrm{A}$ and further assessed by size exclusion chromatography (SEC) (Fig. 4a). For most fusions, the SEC profile showed two major peaks, one representing aggregates and multimers, and one representing the monomer with the corresponding molecular weight of two associated VHH-MG polypeptides. The major portion of the 2L76 VHHMG was found in aggregates; therefore, we excluded it from further analysis (data not shown).

For the remaining six fusions, the fractions corresponding to the monomeric VHH-MG were pooled and further analyzed by SDS-PAGE under both reducing and non-reducing conditions to verify dimerization. Because peaks for monomers and aggregates in the SEC profile slightly overlap, the pooled purified VHH-MGs still contain a small quantity of aggregates. The samples on the non-reducing gel nicely displayed an $80-\mathrm{kDa}$ band (Fig. 4b), which corresponds to a monomeric VHH-MG composed of two VHH-MG polypeptides of $40 \mathrm{kDa}$, as seen in the SDSPAGE under reducing conditions. Moreover, some high-molecular weight bands that most likely represent the aggregates or glycan variants were also observed.

Next, we tested the binding characteristics of the different purified VHH-MG fusions in ELISA and FACS (Fig. 5a and 5b, respectively). A GFP-specific VHH fused to a murine IgG3 (GBP-MG) was used as a negative control [24]. Although all of the six selected fusions showed strong binding on APN-expressing cells, the binding efficiency on the immobilized antigen in ELISA varied a lot, as was observed with the crude samples (Supplementary Fig. S2). These 
findings imply that screening for strong binders via ELISA would not be an ideal approach for applications aiming to target membrane proteins.

Because efficient endocytosis by the target cell population of the potential vaccine-carrying system is of high importance to obtain a strong immune response, we further investigated whether the APN-binding VHH-MG fusions showed specific uptake by cells via the APN receptor. We used APN-expressing BHK21 cells because of their strong binding capacity of VHH-MG in FACS (Fig. 5b). The VHH-MG proteins were stained with a different fluorescent molecule to discriminate between membrane-associated VHH-MG and internalized VHH-MG via the APN receptor. Confocal imaging clearly demonstrated the APN-dependent uptake of VHH-MG fusions, because uptake of irrelevant VHH-MG fusions could not be observed (Fig. 5c). Three VHH-MG fusions (3L94-MG, 2L65-MG and 2L48-MG) were found to be the most potent APN binders and they displayed clear uptake signals only in APN-expressing cells (Fig. 5c).

\subsection{In vivo behavior of selected APN-specific VHH-Fc candidates in a gut ligated loop assay}

To assess the in vivo behavior of the selected VHH-Fc IgG constructs, we performed a gut ligated loop assay in the small intestine (jejunum) of piglets. Figure 6a shows that 3L94-MG and 2L65MG were able to bind to the entire apical epithelium of the villi. In contrast, 2L48 and 2L22 showed a more patchy binding profile. Supplementary Figure $\mathbf{S 4}$ shows that the binding profile is similar in different animals. In addition, Figure 6b, supplementary Figures S5 and S6 show that 3L94MG and 2L65-MG are endocytosed by the enterocytes in a similar way as IMM013. Based on this in vivo behavior in a gut ligated loop assay, we conclude that 3L94-MG and 2L65-MG are the most suitable candidates to target antigens to the small epithelium.

\subsection{Oral immunization with APN-specific VHH-Fc elicits circulating and small intestinal immune responses}

To further demonstrate the potential of the selected VHH-MGs as vaccine delivery system, an oral immunization experiment in piglets was performed and the ensuing systemic and small intestinal immune responses were evaluated through detection of mouse Fc IgG2a-specific serum antibody responses and antibody secreting cells via ELISA and ELIspot, respectively. Figure 7b shows that upon oral administration of 2L65-MG mouse Fc IgG2a-specific $\operatorname{IgG}$ and $\operatorname{IgA}$ serum levels were significantly increased as compared to the control group. Surprisingly, 3L94 did not promote 
mouse Fc IgG2a-specific serum antibody responses. In addition, oral immunization with 2L65MG significantly increased the amount of circulating mouse Fc IgG2a-specific IgA secreting cells at 21 days post primary immunization as compared to day 0 and the control group (Fig. 7c). These results show that APN-specific VHH-MGs promote systemic immunity upon oral administration to piglets. To further show that also intestinal immune responses were enhanced, we evaluated the presence of mouse $\mathrm{Fc} \mathrm{IgG}_{2 \mathrm{a}}$-specific antibody secreting cells in different small intestinal tissues via ELIspot. As shown in Figure 7d, only 2L65-MG significantly enhanced the number of mouse

Fc IgG2a-specific IgA secreting cells in mesenteric lymph nodes and ileal lamina propria and Peyer's patches at 28 dppi. Taken together, these results show that oral administration of 2L65MG triggers systemic and small intestinal immune responses in piglets.

\section{Discussion}

Despite their potential for generating both local and systemic immune responses, only few mucosal vaccines currently exist. The subunit vaccines very often trigger only a weak immunogenic response upon oral delivery, for which tolerance and the lack of uptake and presentation to the APCs are only two of the postulated reasons. Therefore, it was hypothesized that the selective targeting of subunit vaccines to intestinal receptors could help internalization of the vaccine to elicit more effective immune responses. Aminopeptidase $\mathrm{N}$ has previously been described as a receptor for F4 fimbriae that is expressed on a variety of cells, including small intestinal enterocytes and APCs (see ref. [5]), and as a target for oral delivery of mucosal vaccines [6]. Very promising in this respect is that polyclonal antibodies raised against APN in rabbits were shown to be endocytosed by porcine enterocytes after oral administration to young piglets [5]. Moreover, a strong immune response against specific rabbit epitopes of the Fc portion of the polyclonal antibodies was found upon oral administration, whereas no immune response was observed upon oral administration of other polyclonal rabbit antibodies not binding to APN [5]. This led to the hypothesis that APN-dependent targeting of antigens might promote the efficacy of mucosal vaccines, for which a strong APN-binding carrier would be essential [6]. Indeed, targeting of antigen loaded B-glucan particles to APN enhanced their uptake and the immune response against the antigen. 
Here, we demonstrate the isolation and characterization of APN-specific VHHs. VHHs have extensively been used for diagnostic and therapeutic applications because of their peculiar properties [32-34]. Thanks to their small size, VHHs can easily be engineered to make genetic fusions with the model antigens. VHH against MHC class II proteins fused to a tumor-associated antigen has been shown to establish a strong and lasting immune response as a result of efficient processing and presentation of the exogenous antigen by APCs such as dendritic cells, B cells and macrophages [35]. Moreover, targeting an antigen via the Fc domain to Fc $\gamma$ receptors on APCs has been shown to elicit both humoral and cell-mediated immune response (see ref. [36]). Importantly, the Fc domain of an antibody has an added advantage over VHHs specific for Fcy receptors, because they generate bivalent molecules that are often preferred for many applications owing to avidity effects and the capacity to cross-link. Fc fusions also offer many other advantages in terms of improved VHH serum half-life, purification on commercially available resins and recognition by Fc receptors present on the APCs [7, 37]. Thus, the VHHs identified in this study were fused to an Fc domain. We chose to first use the Fc fragment of a known mouse IgG (MG), enabling us to measure a specific immune response against mouse Fc epitopes upon oral delivery of APN-targeted VHH-mouse Fc fusions in pigs. The fact that there are many similarities between pigs and humans in terms of anatomy, physiology and genetics, raises the possibility to use pigs as an effective model system for preclinical studies of human diseases and vaccine efficacy [3840]. However, the Fc domain from murine origin might not display efficient Fc-mediated effector functions in piglets, because, in contrast to the information available about human and mouse Fc receptors, still little is known for pigs, especially on the interaction of the latter with murine $\operatorname{IgG}$ subclasses. It was recently reported that human IgG bind to human ortholog receptors with similar affinities than what is known for the human Fc $\gamma \mathrm{R}$ [41, 42], suggesting that mouse IgG might show a similar binding to porcine Fc $\gamma \mathrm{R}$. However, as long as experimental evidence is lacking on the interaction between murine Fc and porcine Fc receptors, the mouse Fc fragment should be replaced by a porcine $\mathrm{Fc}$ fragment in future experiments with other antigens when using pigs as a model system for in vivo validation of the vaccine targeting strategy.

We further determined the binding of VHH-MG fusions to pAPN via ELISA and to pAPNexpressing cells via flow cytometry. However, because binding to immobilized antigen may not be equivalent to binding to the cell membrane, flow cytometry data was the most reliable. It showed binding to the full-length protein in its native form that is expressed on the biological 
membrane. Moreover, we observed a clear correlation with the binding on intestinal tissue that further confirmed the flow cytometry data. Next, we demonstrated the uptake of VHH-MGs on pAPN-expressing cells and porcine intestinal tissue in a gut ligated loop experiment. These data support the efficient transport of the APN-targeted VHH-MGs across the small intestinal epithelium. At least for one VHH-MG this endocytosis resulted in the induction of robust antigenspecific systemic and small intestinal IgA responses upon oral administration to piglets. Why endocytosis of the other VHH-MG did not trigger immune responses warrants further investigation. This discrepancy between internalisation and induction of immune responses might be explained by the SISP model itself. Although this system represents one of the best options to assess targeting and uptake across the small intestinal epithelium in a large animal model with some degree of peristalsis still occuring, it does not take into account the impact of bile salts and transit of food on this internalization. Moreover, animals are anesthetized, which might influence gut homeostasis and thus internalization of the APN-targeted VHH-MGs.

Orally delivered subunit vaccines have to pass the gut epithelia that is protected by mucosal immune defense mechanisms and well-developed barriers such as low gastric $\mathrm{pH}$ and the presence of proteolytic enzymes resulting in the degradation of vaccine materials. Thus, oral delivery of VHH-based subunit vaccines might not generate a strong systemic immune response that would otherwise be true when injected. To circumvent these hurdles, the subunit vaccine can be protected by encapsulation in a biodegradable particulate delivery system. Baert et al. reported that oral administration of $\beta$-glucan microparticles conjugated to mAb against APN (IMM013) can efficiently deliver the antigen payload and elicits a stronger immune response in piglets [6].

\section{Conclusion}

In conclusion, we have identified pAPN-specific VHHs, fused them to the Fc domain of a conventional antibody and showed their efficient endocytosis by the small intestinal epithelium under physiological conditions and their ability to elicit systemic and mucosal antibody responses. The conjugation of a clinically relevant antigen to these pAPN-specific VHHs can be envisioned as a next step to evaluate whether delivery of vaccine antigens to APN on small intestinal enterocytes can trigger protective immune response at the gut epithelium. Finally, the present study extends the use of $\mathrm{VHH}$ as a delivery vehicle, thereby paving the way to develop a more rational 
approach to the broader basket of effective vaccine delivery systems to promote human and animal health.

\section{Conflict of interests}

We declare that we have no financial and personal relationships with other people or organizations that can inappropriately influence our work and there is no conflict of interest regarding the publication of this paper.

\section{Author Contributions}

A.D., E.C., B.D. and V.V. conceived the idea and designed the research; S.B., A.T. and S.P. performed cloning, production, purification and characterization; S.B., H.W. and A.T. performed cell-based experiments; R.S. performed gut-ligated loop experiments and the in vivo experiment; P.J. designed and obtained the yeast cloning vector adapted for GoldenBraid cloning strategy; S.B., H.W., A.T., R.S., A.D., B.D. and E.C. analyzed the data; S.B. and A.D. wrote the manuscript with contributions from R.S., H.W. and B.D.; All authors reviewed the manuscript before submission.

\section{Acknowledgments}

The authors would like to thank Annick Bleys for assistance with the manuscript preparation and Dr. Thomas Jacobs for assisting in designing and setting up the Gibson cloning strategy on BioXP. S.B., A.T., S.P., H.W. and R.S. are recipients of a fellowship from the Ghent University (BOF15/GOA/031). This work was supported by institutional funding and support from Ghent University and VIB, and a research grant from the Research Foundation Flanders (G0C9714N); P.J was supported by the European Commission (H2020-MSCA-IF-2014 Proposal 658701ImmunoFarm). Bert Devriendt holds a postdoctoral grant of the Research Foundation Flanders (FWO-Vlaanderen).

\section{References}

[1] P. Juarez, V. Virdi, A. Depicker, D. Orzaez, Biomanufacturing of protective antibodies and other therapeutics in edible plant tissues for oral applications, Plant Biotechnol. J., 14 (2016) 1791-1799.

[2] N. Lycke, Recent progress in mucosal vaccine development: potential and limitations, Nat. Rev. Immunol., 12 (2012) 592-605. 
[3] V. Snoeck, W. Van den Broeck, V. De Colvenaer, F. Verdonck, B. Goddeeris, E. Cox, Transcytosis of F4 fimbriae by villous and dome epithelia in F4-receptor positive pigs supports importance of receptordependent endocytosis in oral immunization strategies, Vet. Immunol. Immunopathol., 124 (2008) 29-40.

[4] B. Devriendt, B.G. De Geest, B.M. Goddeeris, E. Cox, Crossing the barrier: Targeting epithelial receptors for enhanced oral vaccine delivery, J. Control. Release, 160 (2012) 431-439.

[5] V. Melkebeek, K. Rasschaert, P. Bellot, K. Tilleman, H. Favoreel, D. Deforce, B.G. De Geest, B.M. Goddeeris, E. Cox, Targeting aminopeptidase N, a newly identified receptor for F4ac fimbriae, enhances the intestinal mucosal immune response, Mucosal Immunol., 5 (2012) 635-645.

[6] K. Baert, B.G. de Geest, R. de Rycke, A.B. da Fonseca Antunes, H. de Greve, E. Cox, B. Devriendt, $\beta$ glucan microparticles targeted to epithelial APN as oral antigen delivery system, J. Control. Release, 220 (2015) 149-159.

[7] B. Narasimhan, J.T. Goodman, J.E. Vela Ramirez, Rational design of targeted next-generation carriers for drug and vaccine delivery, Annu. Rev. Biomed. Eng., 18 (2016) 25-49.

[8] W.W. Cheng, T.M. Allen, The use of single chain Fv as targeting agents for immunoliposomes: an update on immunoliposomal drugs for cancer treatment, Expert Opin. Drug Deliv., 7 (2010) 461-478.

[9] C.R. Robinson, R.T. Sauer, Optimizing the stability of single-chain proteins by linker length and composition mutagenesis, Proc. Natl. Acad. Sci. USA, 95 (1998) 5929-5934.

[10] J.N. Duarte, J.J. Cragnolini, L.K. Swee, A.M. Bilate, J. Bader, J.R. Ingram, A. Rashidfarrokhi, T. Fang, A. Schiepers, L. Hanke, H.L. Ploegh, Generation of immunity against pathogens via single-domain antibody-antigen constructs, J. Immunol., 197 (2016) 4838-4847.

[11] T. De Meyer, S. Muyldermans, A. Depicker, Nanobody-based products as research and diagnostic tools, Trends Biotechnol., 32 (2014) 263-270.

[12] J. Helma, M.C. Cardoso, S. Muyldermans, H. Leonhardt, Nanobodies and recombinant binders in cell biology, J. Cell Biol., 209 (2015) 633-644.

[13] S. Muyldermans, Nanobodies: natural single-domain antibodies, Annu. Rev. Biochem., 82 (2013) 775797.

[14] R.W. Ellis, R.J. Gerety, Key issues in the selection of an expression system for vaccine antigens, J. Med. Virol., 31 (1990) 54-58.

[15] A. Frenzel, M. Hust, T. Schirrmann, Expression of recombinant antibodies, Front. Immunol., 4 (2013) 217.

[16] V. Yusibov, N. Kushnir, S.J. Streatfield, Antibody production in plants and green algae, Annu. Rev. Plant Biol., 67 (2016) 669-701.

[17] F.R. Schmidt, Recombinant expression systems in the pharmaceutical industry, Appl. Microbiol. Biotechnol., 65 (2004) 363-372.

[18] D. Weinacker, C. Rabert, A.B. Zepeda, C.A. Figueroa, A. Pessoa, J.G. Farías, Applications of recombinant Pichia pastoris in the healthcare industry, Braz. J. Microbiol., 44 (2013) 1043-1048.

[19] J.M. Cregg, J.L. Cereghino, J. Shi, D.R. Higgins, Recombinant protein expression in Pichia pastoris, Mol. Biotechnol., 16 (2000) 23-52.

[20] R.E. Sallach, V.P. Conticello, E.L. Chaikof, Expression of a recombinant elastin-like protein in Pichia pastoris, Biotechnol. Prog., 25 (2009) 1810-1818.

[21] M. Donini, C. Marusic, Current state-of-the-art in plant-based antibody production systems, Biotechnol. Lett., 41 (2019) 335-346.

[22] C. Lundqvist, M.-L. Hammarström, L. Athlin, S. Hammarström, Isolation of functionally active intraepithelial lymphocytes and enterocytes from human small and large intestine, J. Immunol. Methods, 152 (1992) 253-263.

[23] E. Pardon, T. Laeremans, S. Triest, S.G.F. Rasmussen, A. Wohlkönig, A. Ruf, S. Muyldermans, W.G.J. Hol, B.K. Kobilka, J. Steyaert, A general protocol for the generation of Nanobodies for structural biology, Nat. Protoc., 9 (2014) 674-693.

[24] T. De Meyer, B. Laukens, J. Nolf, E. Van Lerberge, R. De Rycke, A. De Beuckelaer, S. De Buck, N. Callewaert, A. Depicker, Comparison of VHH-Fc antibody production in Arabidopsis thaliana, Nicotiana benthamiana and Pichia pastoris, Plant Biotechnol. J., 13 (2015) 938-947. 
[25] A. Coddens, M. Loos, D. Vanrompay, J.P. Remon, E. Cox, Cranberry extract inhibits in vitro adhesion of $\mathrm{F} 4$ and $\mathrm{F}_{18}{ }^{+}$Escherichia coli to pig intestinal epithelium and reduces in vivo excretion of pigs orally challenged with F18 ${ }^{+}$verotoxigenic E. coli, Vet. Microbiol., 202 (2017) 64-71.

[26] B. Devriendt, M. Gallois, F. Verdonck, Y. Wache, D. Bimczok, I.P. Oswald, B.M. Goddeeris, E. Cox, The food contaminant fumonisin $\mathrm{B}(1)$ reduces the maturation of porcine CD11R1(+) intestinal antigen presenting cells and antigen-specific immune responses, leading to a prolonged intestinal ETEC infection, Vet Res, 40 (2009) 40.

[27] E. De Genst, F. Handelberg, A. Van Meirhaeghe, S. Vynck, R. Loris, L. Wyns, S. Muyldermans, Chemical basis for the affinity maturation of a camel single domain antibody, J. Biol. Chem., 279 (2004) 53593-53601.

[28] K.E. Conrath, M. Lauwereys, M. Galleni, A. Matagne, J.-M. Frère, J. Kinne, L. Wyns, S. Muyldermans, $\beta$-Lactamase inhibitors derived from single-domain antibody fragments elicited in the Camelidae, Antimicrob. Agents Chemother., 45 (2001) 2807-2812.

[29] V. Virdi, A. Coddens, S. De Buck, S. Millet, B.M. Goddeeris, E. Cox, H. De Greve, A. Depicker, Orally fed seeds producing designer IgAs protect weaned piglets against enterotoxigenic Escherichia coli infection, Proc. Natl. Acad. Sci. USA, 110 (2013) 11809-11814.

[30] K. Moonens, M. De Kerpel, A. Coddens, E. Cox, E. Pardon, H. Remaut, H. De Greve, Nanobody mediated inhibition of attachment of F18 fimbriae expressing Escherichia coli, PLoS ONE, 9 (2014) e114691.

[31] S. Bakshi, A. Depicker, B. Schepens, X. Saelens, P. Juarez, A two-amino acid mutation in murine IgA enables downstream processing and purification on staphylococcal superantigen- like protein 7, J. Biotechnol., 294 (2019) 26-29.

[32] P. Lafaye, T. Li, Use of camel single-domain antibodies for the diagnosis and treatment of zoonotic diseases, Comp. Immunol. Microbiol. Infect. Dis., 60 (2018) 17-22.

[33] S. Steeland, R.E. Vandenbroucke, C. Libert, Nanobodies as therapeutics: big opportunities for small antibodies, Drug Discov. Today, 21 (2016) 1076-1113.

[34] I. Van Audenhove, J. Gettemans, Nanobodies as versatile tools to understand, diagnose, visualize and treat cancer, EBioMedicine, 8 (2016) 40-48.

[35] T. Fang, C.H.M.J. Van Elssen, J.N. Duarte, J.S. Guzman, J.S. Chahal, J. Ling, H.L. Ploegh, Targeted antigen delivery by an anti-class II MHC VHH elicits focused $\alpha$ MUC1(Tn) immunity, Chem. Sci., 8 (2017) 5591-5597.

[36] D.M. Czajkowsky, J. Hu, Z.F. Shao, R.J. Pleass, Fc-fusion proteins: new developments and future perspectives, EMBO Mol. Med., 4 (2012) 1015-1028.

[37] F. Nimmerjahn, J.V. Ravetch, Antibodies, Fc receptors and cancer, Curr. Opin. Immunol., 19 (2007) 239-245.

[38] J. Guo, F. Li, Q. He, H. Jin, M. Liu, S. Li, S. Hu, Y. Xiao, D. Bi, Z. Li, Neonatal Fc receptor-mediated IgG transport across porcine intestinal epithelial cells: potentially provide the mucosal protection, DNA Cell Biol., 35 (2016) 301-309.

[39] F. Meurens, A. Summerfield, H. Nauwynck, L. Saif, V. Gerdts, The pig: a model for human infectious diseases, Trends Microbiol., 20 (2012) 50-57.

[40] R.J. Ober, C.G. Radu, V. Ghetie, E.S. Ward, Differences in promiscuity for antibody-FcRn interactions across species: implications for therapeutic antibodies, Int. Immunol., 13 (2001) 1551-1559.

[41] G. Dekkers, A.E.H. Bentlage, T.C. Stegmann, H.L. Howie, S. Lissenberg-Thunnissen, J. Zimring, T. Rispens, G. Vidarsson, Affinity of human IgG subclasses to mouse Fc gamma receptors, Mabs-Austin, 9 (2017) 767-773.

[42] J. Egli, T. Schlothauer, C. Spick, S. Seeber, T. Singer, A. Odermatt, A. Iglesias, The Binding of Human IgG to Minipig FcRs - Implications for Preclinical Assessment of Therapeutic Antibodies, Pharm. Res., 36 (2019). 
a

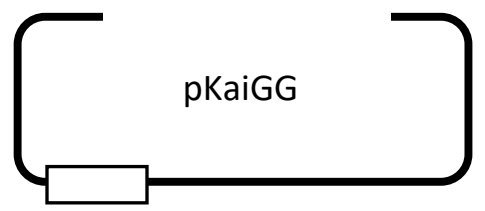

Sapl linearized vector

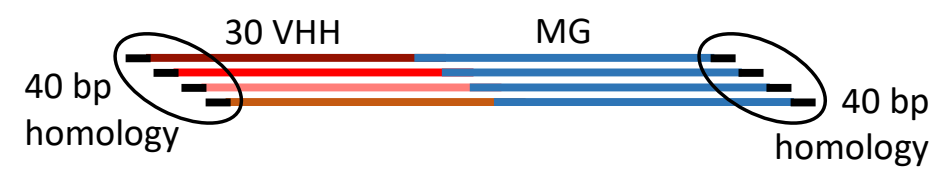

DNA sequence, received with the BioXP kit
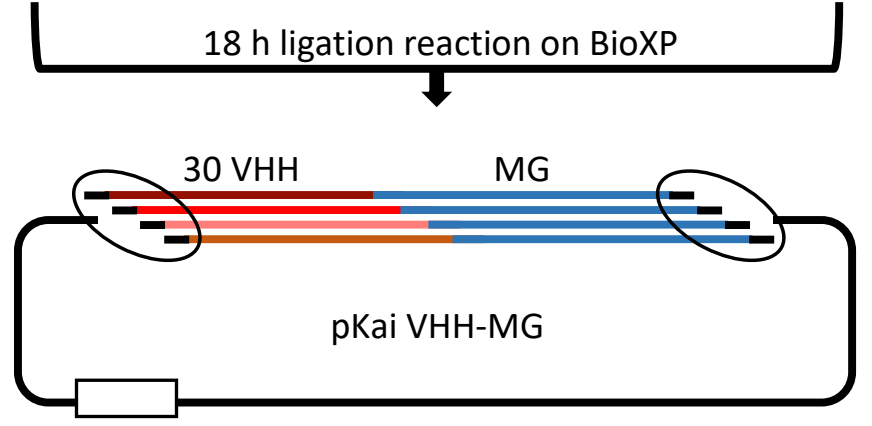

b

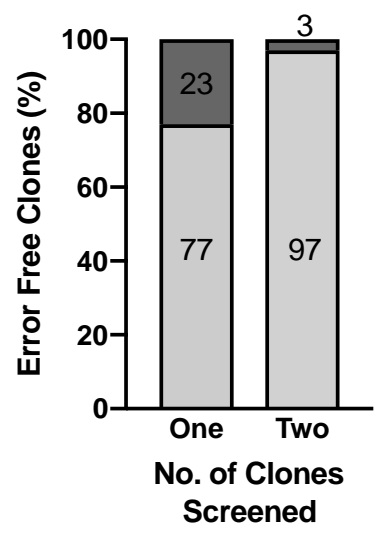

Figure 1. Schematic representation of the BioXp ${ }^{\mathrm{TM}} 3200$ System-based Gibson cloning strategy to efficiently obtain VHH-MG fusions (a). The $40 \mathrm{bp}$ at the ends of the VHH-MG fusion fragments are homologous with the ends of the SapI-linearized pKaiGG vector. MG represents the Fc domain of Mouse IgG. VHH-MG fragments were synthesized and ligated into SapI-digested pKaiGG in an overnight run on the BioXP system. DH5 $\alpha$ cells were transformed with ligation samples and transformants were screened on Zeocin (Zeo)-supplemented medium. Subsequently, DNA was isolated from four randomly selected clones and analyzed by restriction digestion or colony PCR and sequencing to screen for positive clones. (b) Efficiency of obtaining error-free clones via the BioXP cloning system. The probability to obtain errorfree clones was $77 \%$ when one clone was screened and $97 \%$ when two clones were screened per construct via sequencing. 
a
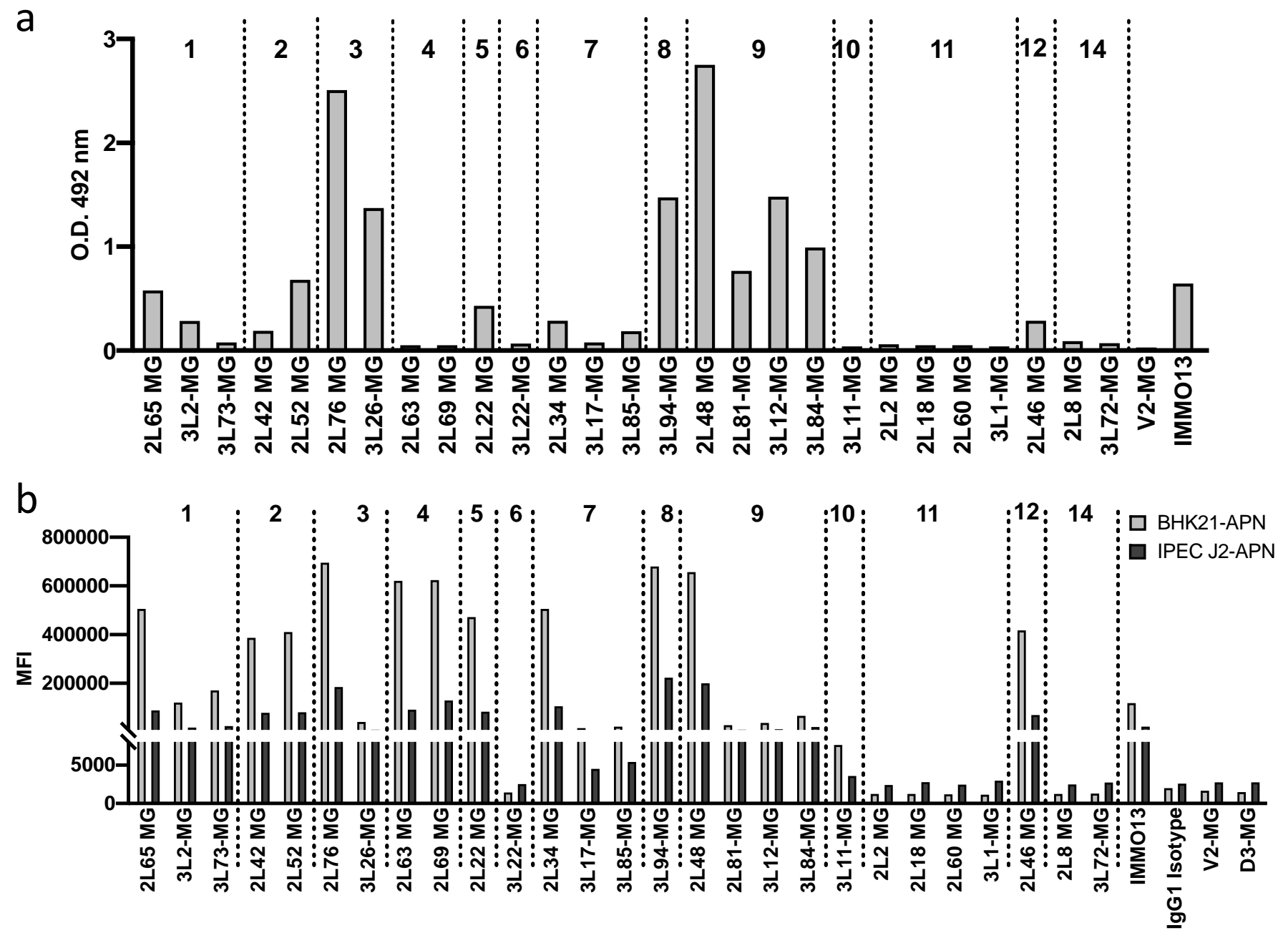

Figure 2. Selection of APN-specific VHH-MG via ELISA and flow cytometry. Clones are arranged according to the family they belong to and separated by a dotted line. (a) ELISA plates were coated with APN and incubated with the culture medium. APN binding of VHH-MG is depicted as O.D. values of the colorimetric reaction. (b) Flow cytometry screening for the VHH-MGs that bind to full-length APN expressed on the membrane of APN-transfected BHK21 (BHK21-APN, gray bars) and APN-transfected IPECJ2 (IPEC-J2-APN, black bars). IMM013 (murine IgG1) is the positive control. IgG1 isotype, V2-MG and D3MG are the negative controls. V2-MG and D3-MG fusions contain a VHH (V2 and D3, respectively) that binds an irrelevant target. The graph shows the mean fluorescence intensity (MFI). 

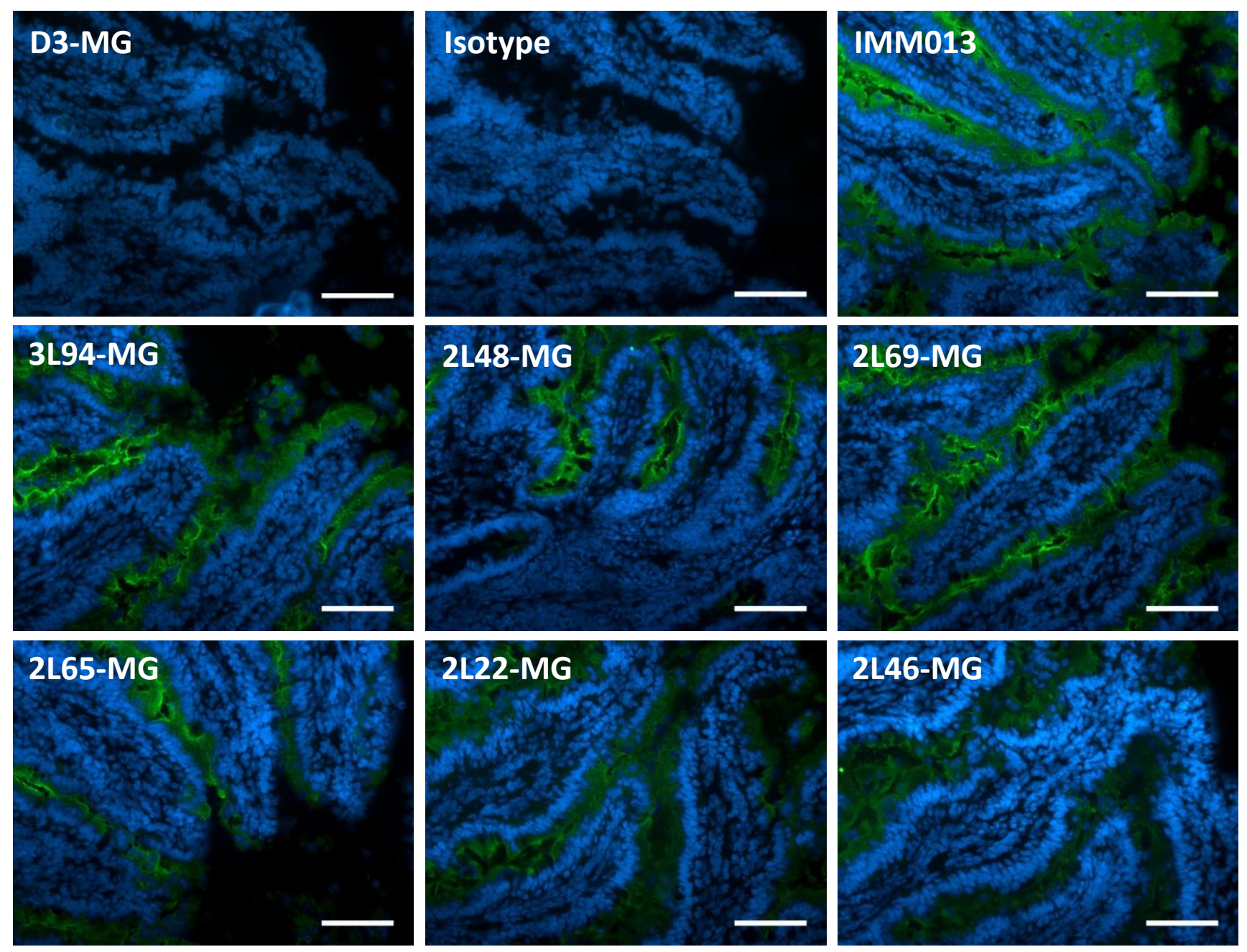

Figure 3. Binding analysis of anti-APN VHH-MG fusions on small intestinal tissue. Equal quantities of VHH-MG obtained after induction in yeast culture medium were assessed for binding on intestinal ileum. IMM013 is a positive control, and Isotype and D3-MG are the negative controls. Binding of the VHH-Fc fusions to the tissue was visualized by anti-mouse IgG-FITC (green) and counterstained with Hoechst (blue); scale bar: $100 \mu \mathrm{m}$. 
a
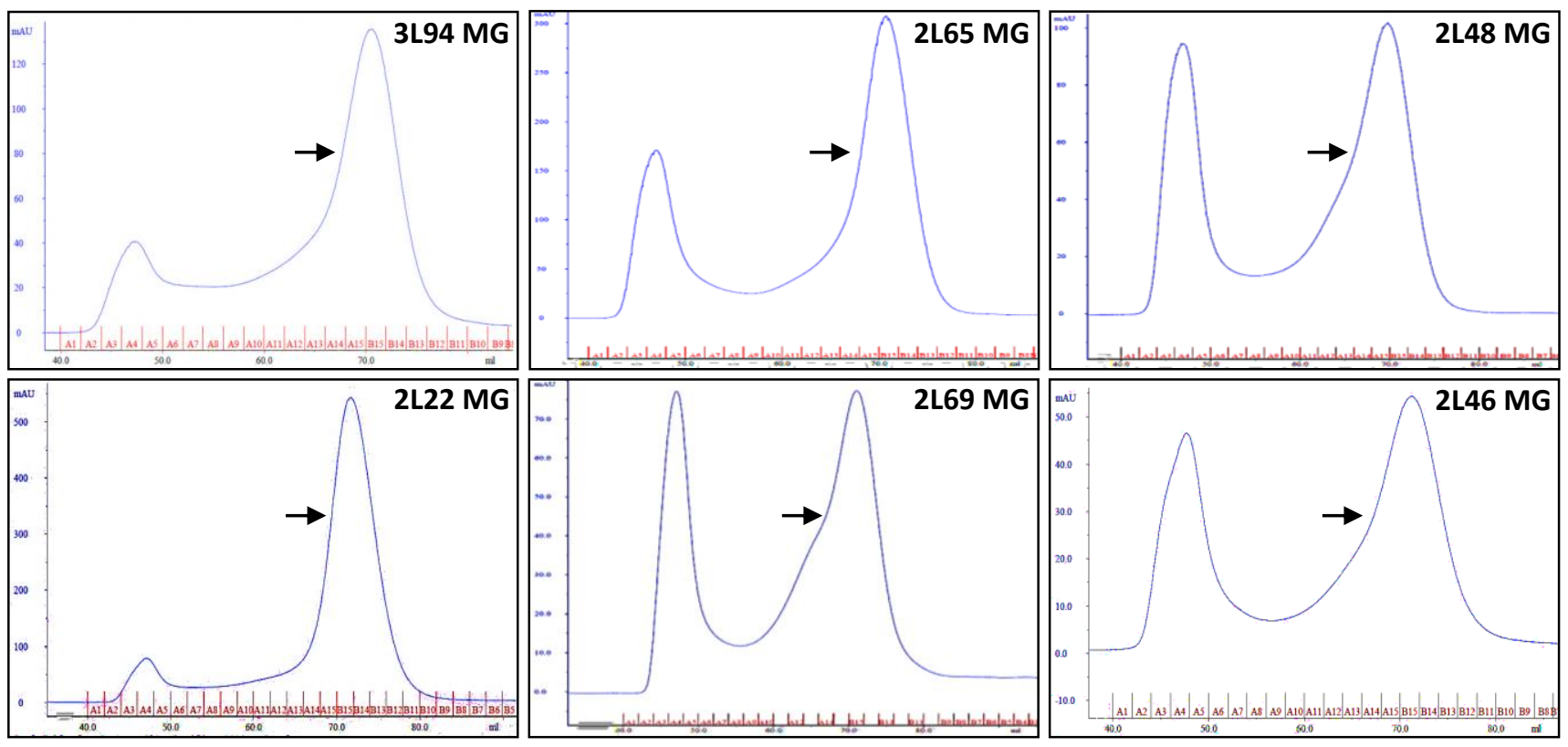

b
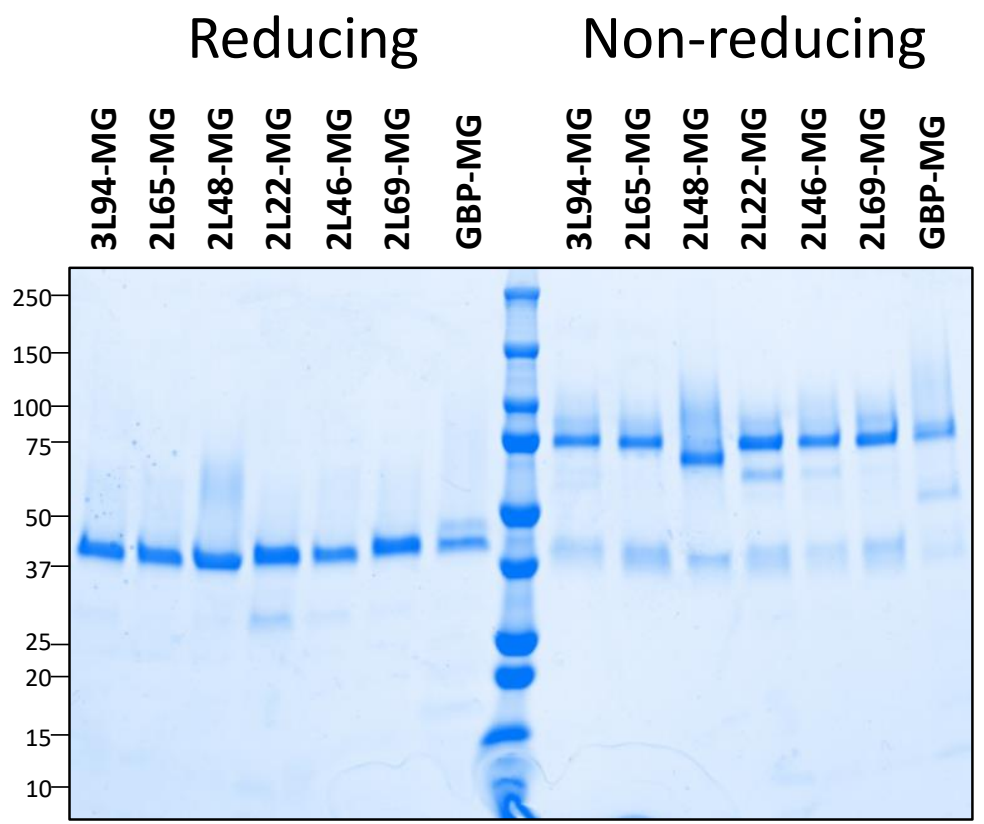

Figure 4. Purification of the different VHH-MG fusions. (a) Size exclusion chromatography (SEC) profile of six different VHH-MG fusions after protein A purification, labeled with its respective name at the top right corner. The arrows indicate the position and molecular weight of $\sim 80 \mathrm{kDa}$, corresponding to 
monomeric VHH-MG fusions assembled by disulfide bridges between two VHH-MG polypeptides ( 40 $\mathrm{kDa}$ each). (b) SDS-PAGE analysis of pooled fractions of the $80-\mathrm{kDa}$ peak after SEC on 4-20\% polyacrylamide gel under both reducing (left) and non-reducing (right) conditions. The expected position of the intact full-length polypeptide is indicated by the arrowhead for monovalent VHH-MG ( 40 kDa) in reducing conditions and by the arrow for bivalent VHH-MG $(\sim 80 \mathrm{kDa})$ in non-reducing conditions. M, molecular weight marker $(\mathrm{kDa})$.

(Yield- 3L94-MG: 2.7 mg/L; 2L48-MG: 2.3 mg/L; 2L69-MG: 1.9 mg/L; 2L65-MG: 5.9 mg/L; 2L22-MG: $8 \mathrm{mg} / \mathrm{L}$; 2L46-MG: $1.4 \mathrm{mg} / \mathrm{L})$. 
a

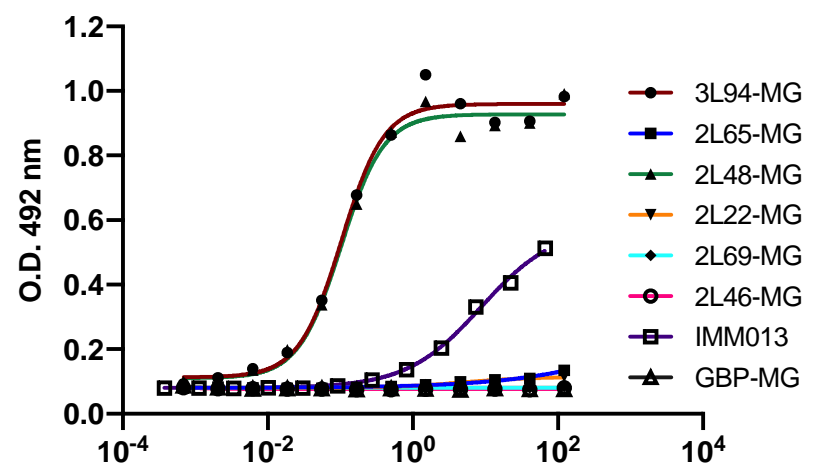

b

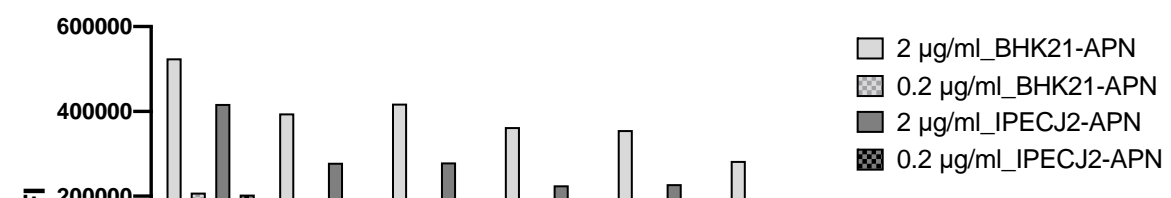

C
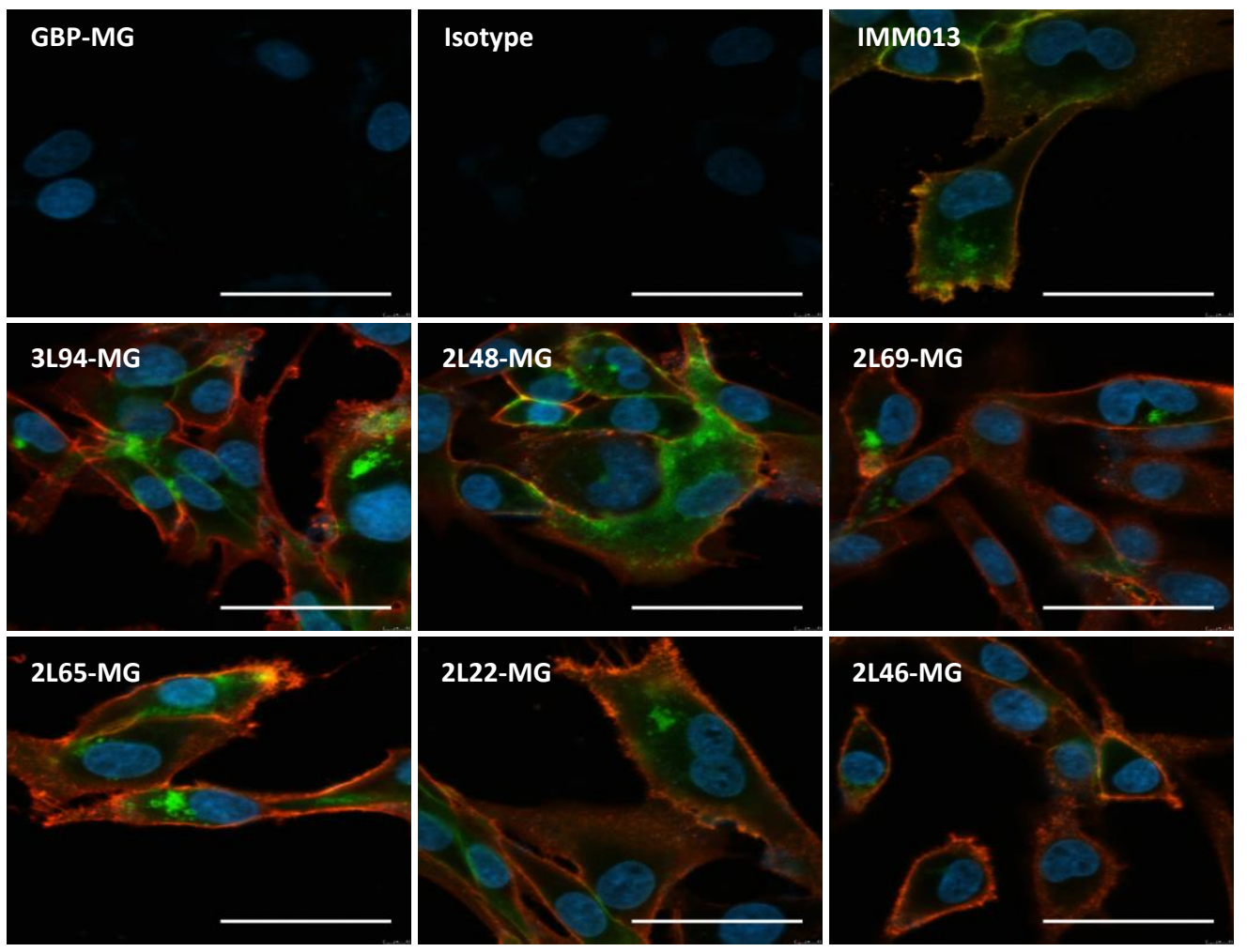
Figure 5. Binding analysis and APN-mediated endocytosis of purified VHH-MG fusions. (a) APNbinding ELISA with purified VHH-MG fusions. A three-fold dilution series of purified VHH-MGs were incubated on an APN-coated microtiter plate and probed with anti-mouse IgG conjugated with horse radish peroxidase. The binding of VHH-MG to immobilized antigen is depicted as OD (492 nm) values. IMM013 is a positive and GBP-MG is a negative control. The background threshold was determined as twice the OD (492 nm) value of the negative control. (b) Binding of the VHH-MG fusions to APN-expressing cells determined by flow cytometry. Graph shows the number of APN-positive cells that bind to VHH-MG at the indicated concentration $(\mu \mathrm{g} / \mathrm{ml})$. (c) Endocytosis of VHH-MG by APN-expressing cell lines. APNexpressing cells were incubated with VHH-MG on ice to allow binding followed by incubation at $37^{\circ} \mathrm{C}$ for 30 min to allow endocytosis. Cells were then fixed and stained with AF568-conjugated anti-mouse IgG (red) to visualize bound VHH-MG. Following permeabilization, cells were stained with anti-mouse IgGFITC (green) to visualize endocytosed VHH-MG. Nucleus was stained with Hoechst (blue). IMM013 is the positive, and isotype and GBP-MG are the negative controls. Images represent a single confocal $\mathrm{z}$ section, scale bars: $100 \mu \mathrm{m}$. 


\section{Figure 6}

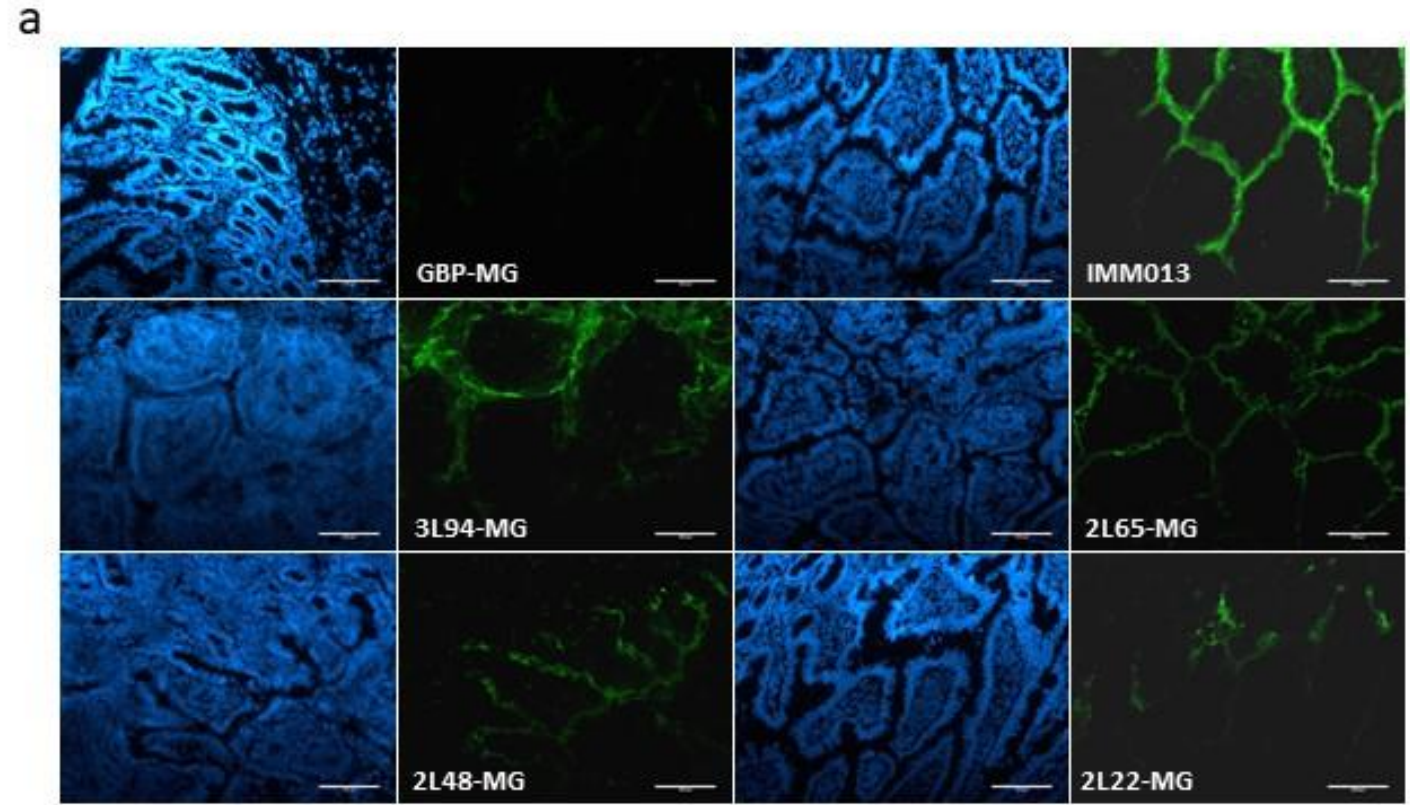

b
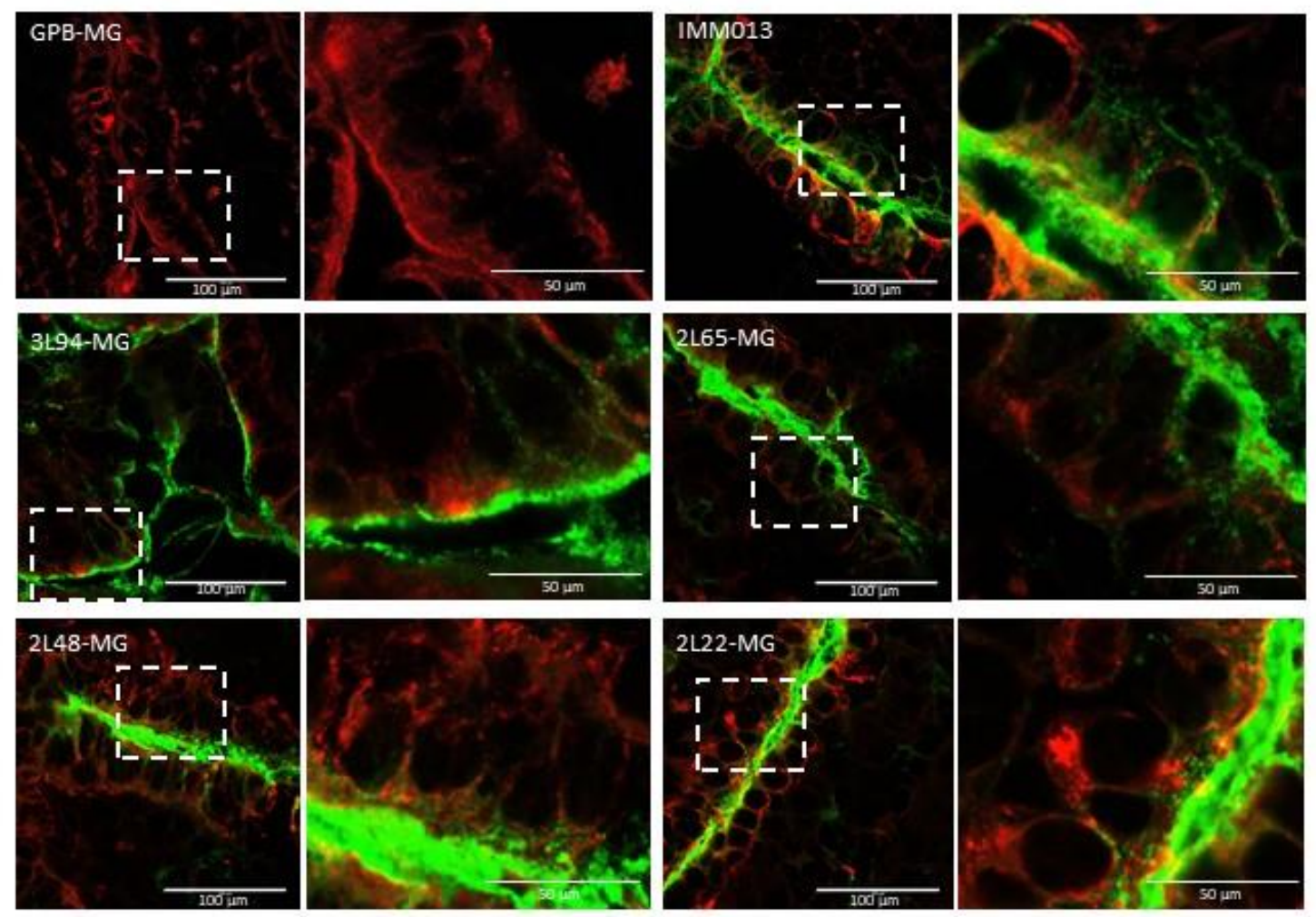
Figure 6. Gut ligated loop experiment to determine the uptake of VHH-MG by enterocytes. (a) Sections from jejunal loops stained with sheep anti-mouse IgG (FITC, green) and counterstained with Hoechst (blue). The different anti-APN VHH-MG and anti-APN mAb (IMM013) are detected on the epithelial cells of the villi. (b) Sections from jejunal loops stained with sheep anti-mouse IgG-FITC (green) and counterstained with anti-cytokeratin-TexasRed (red). Confocal images with representative zooms (square boxes) show the uptake of VHH-MGs and IMM013 by small intestinal enterocytes. Representative of three independent experiments. (scale bars: $100 \mu \mathrm{m}$; scale bars for zoom: $50 \mu \mathrm{m}$ ). 
Figure 7

a

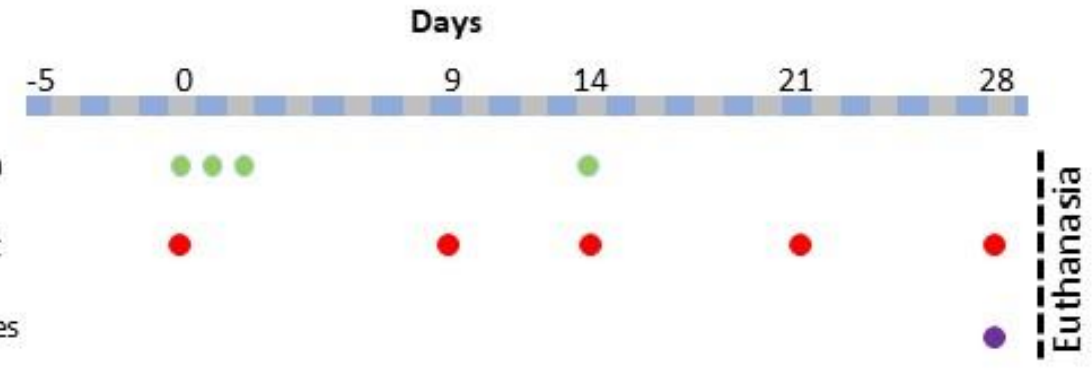

b

IgG
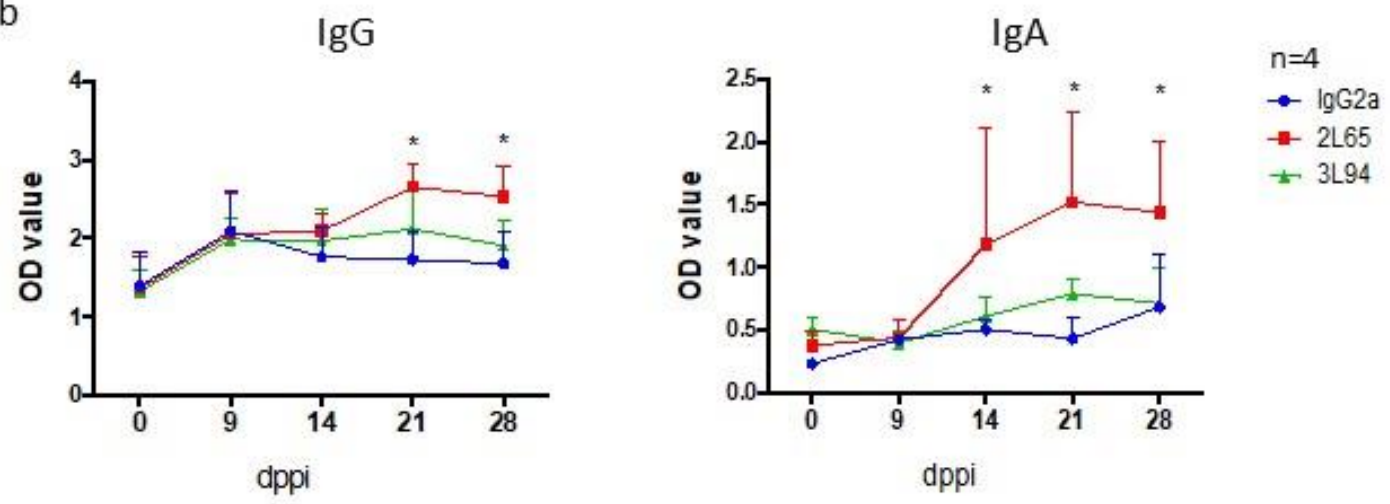

C
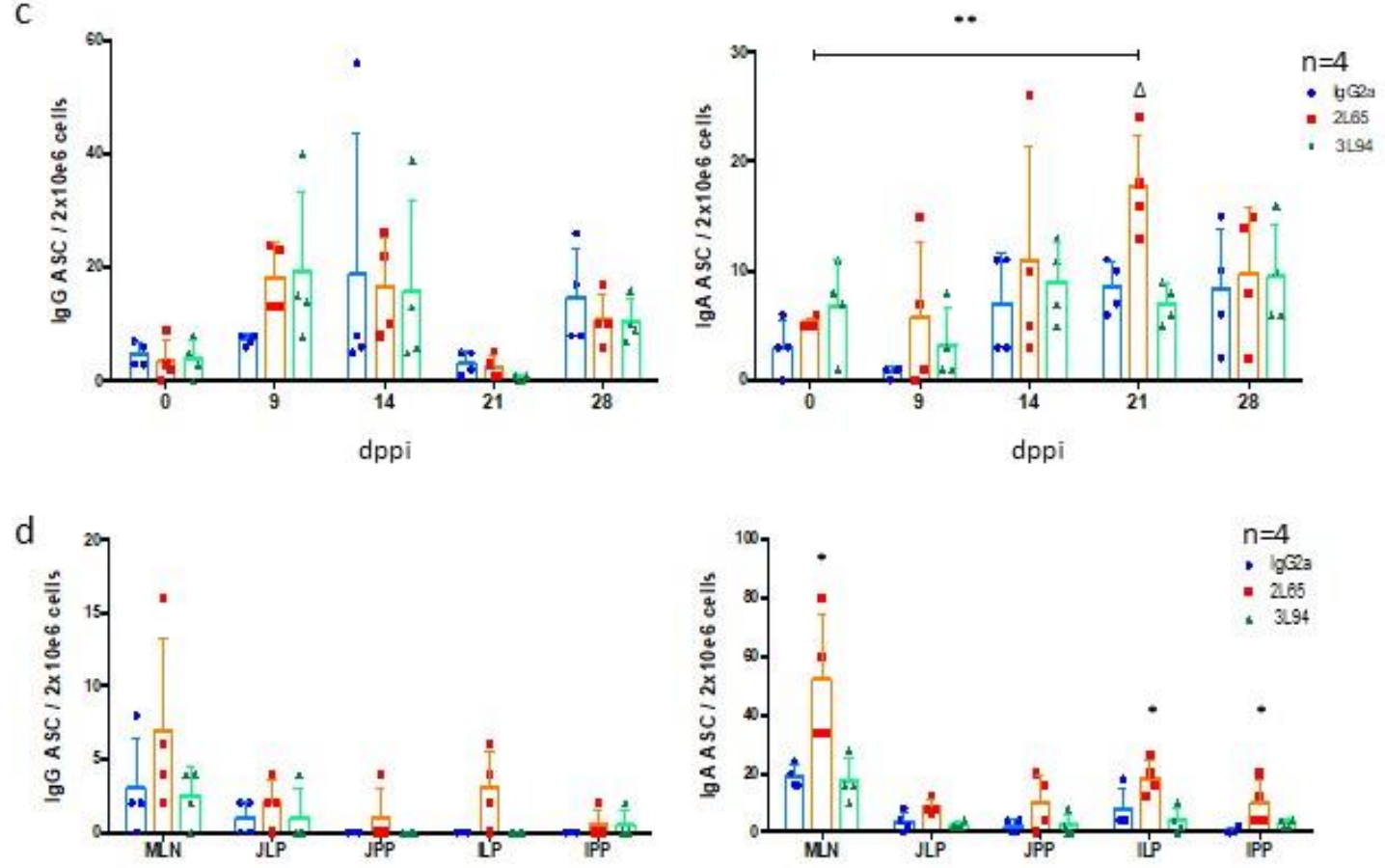
Figure 7. Oral immunization of piglets with VHH-MG triggers circulating and small intestinal antibody responses. (a) design of the oral immunization experiment. (b) mouse IgG2a-specific serum IgG and IgA responses upon oral immunization of piglets (n=4/group) with $1 \mathrm{mg}$ VHH-MG 2L65 and 3L94 and an equimolar amount irrelevant mouse IgG2a. dppi: days post primary immunization; OD: optical density. Data are presented as the mean $+\mathrm{sd}$. *, p $<0.05$ to IgG2a (Friedman). (c) the amount of circulating mouse IgG2a-specific IgG and IgA secreting cells at the indicating time points. ASC: antibody secreting cells. **, $\mathrm{p}<0.01$ to day $0 ; \Delta, \mathrm{p}<0.05$ to IgG2a (Friedman). (d) the amount of small intestinal mouse IgG2a-specific IgG and IgA secreting cells at d28 post primary immunization. MLN: mesenteric lymph nodes; JLP: jejunal lamina propria; JPP: jejunal Peyer's patches; ILP: ileal lamina propria; IPP: ileal Peyer's patches. *, $p<0.05$ (Holm-Sidak). 


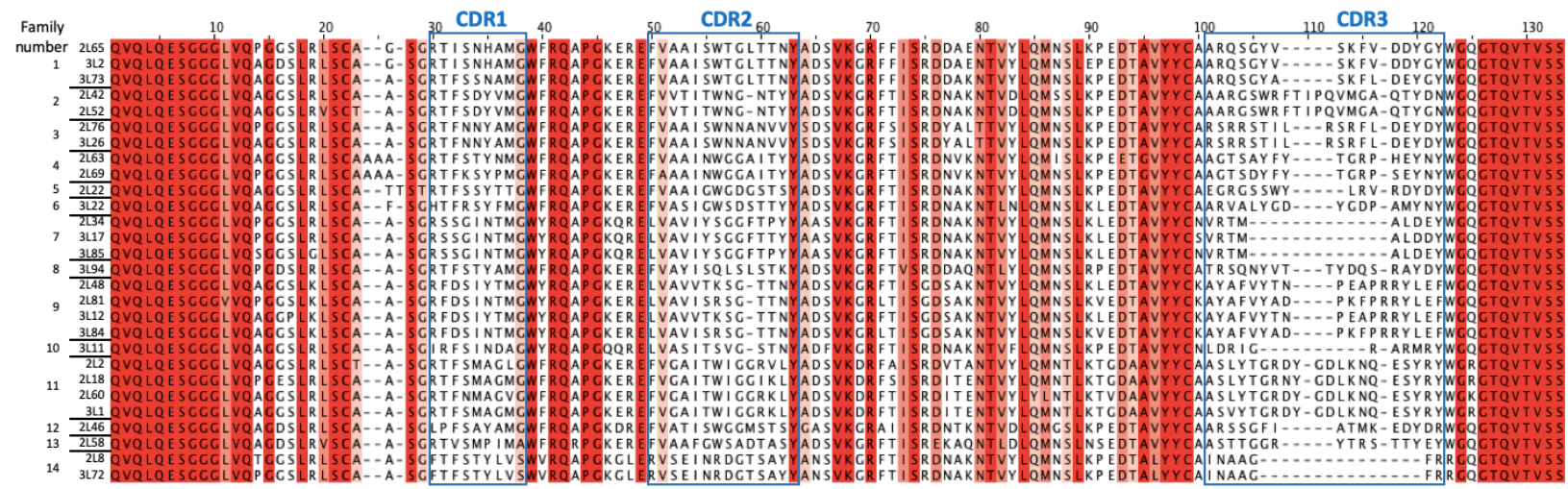

Supplementary Figure S1. Sequence alignment of the 28 anti-APN VHHs. The VHH alignment was carried out with the T-Coffee program (http://www.ebi.ac.uk/) (amino acid sequences). The complementarity-determining regions (CDRs) were predicted using an online tool (http://ofranservices.biu.ac.il/site/services/paratome/index.html) and are boxed (CDR1, CDR2, CDR3). The different family classifications are indicated on the left-hand side of the alignment. Amino acid numbering is indicated on the top of the alignment.

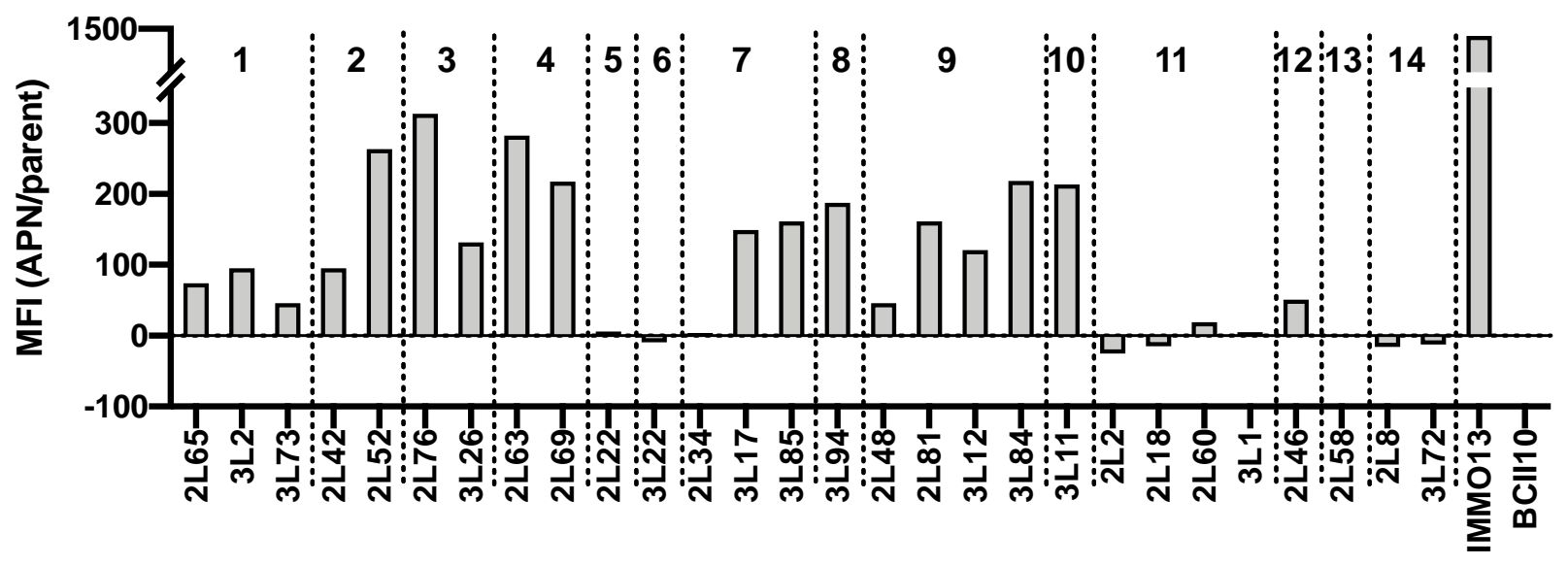

Supplementary Figure S2. Screening of VHHs that bind APN-expressing cell lines. Crude periplasmic extracts were prepared from TG1 cells harboring recombinant phagemids from the library. The 28 APNbinding VHH candidates were screened on APN-expressing cell lines by flow cytometry. The clones were grouped according to their similarity in CDR3 sequence; numbers on top between the dotted lines refer to the family to which the different clones belong. The Y-axis represents the ratio of median fluorescence intensity (MFI) of APN-transfected (APN) and parental non-transfected BHK21 cells (parent). 


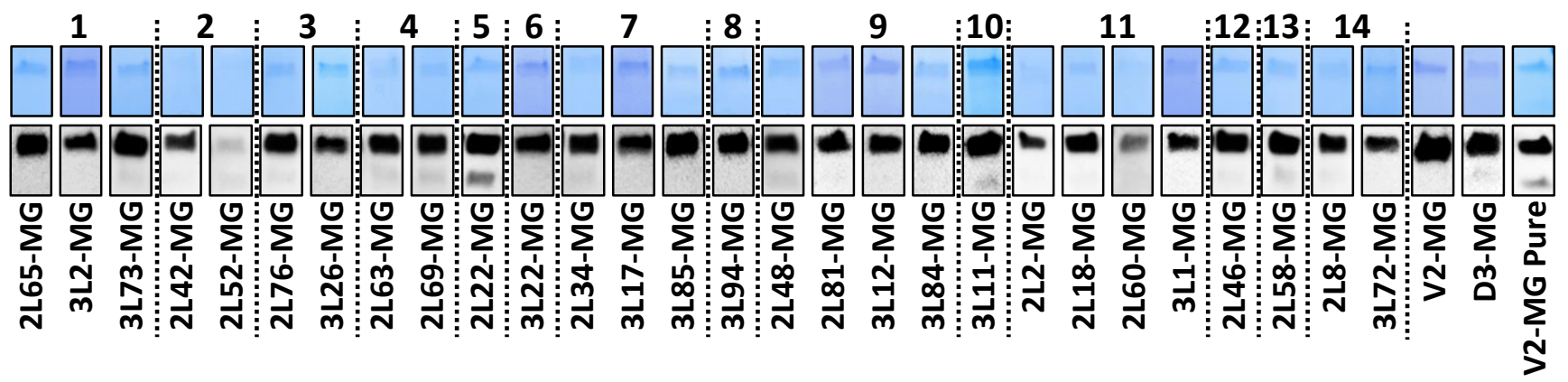

Supplementary Figure S3. Expression of different variants of APN-specific VHH-MG fusions in Pichia pastoris. The culture medium of yeast transformants (4 colonies for each construct) were screened by Coomassie staining and western blot analysis to identify the highest expressing clone. Here, only one high expressing clone for each construct is shown and they were grouped according to their CDR3 similarity; the numbers between the dotted lines represent the family to which they belong. Protein expression was induced for $48 \mathrm{~h}$ with $1 \%$ methanol. Cultures were harvested and supernatant was analyzed by SDS-PAGE (Top) and western blot (Bottom). 'V2-MG Pure' was used as a loading control and $500 \mathrm{ng}$ of samples were loaded for Coomassie staining and $200 \mathrm{ng}$ for western blot. 
Pig 1

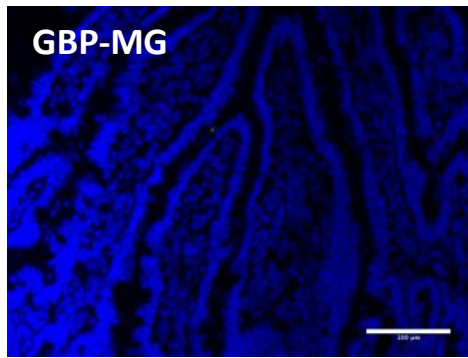

\section{L22-MG}
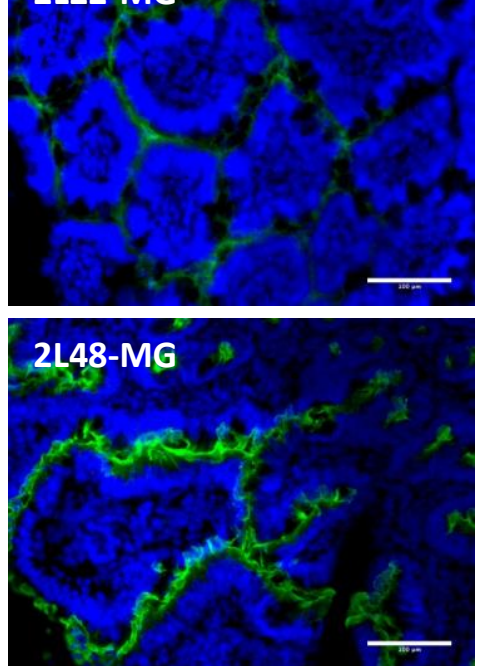

\section{L65-MG}
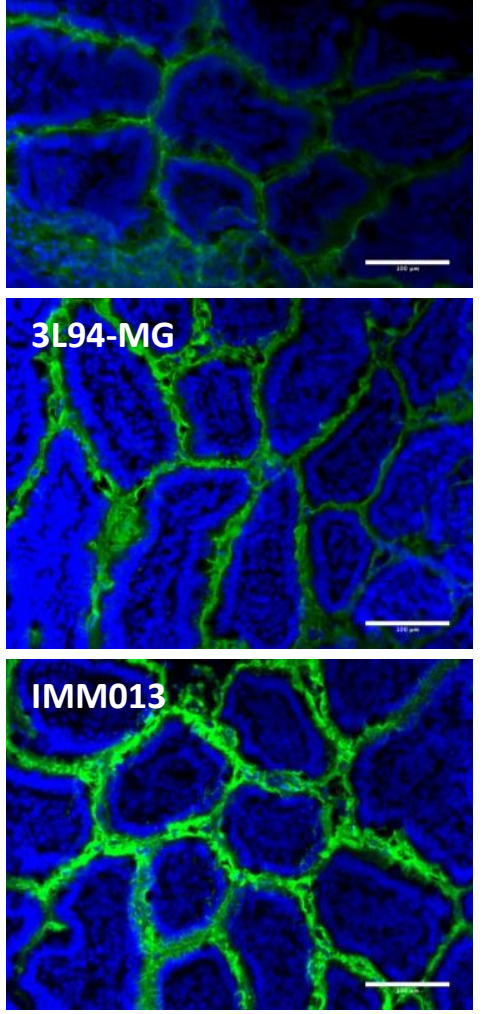

Pig 2
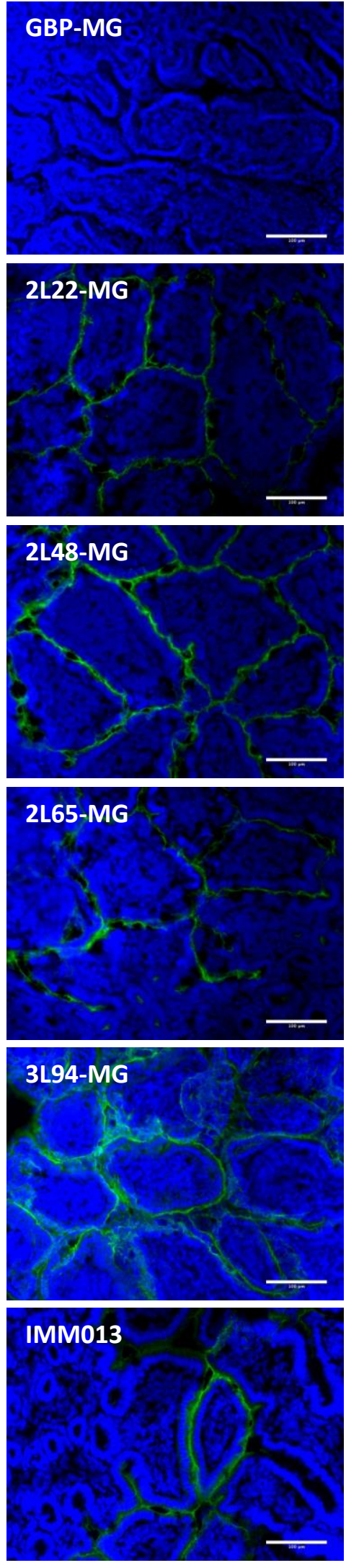

Pig 3

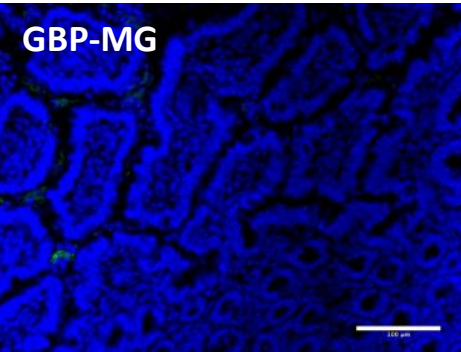

2L22-MG
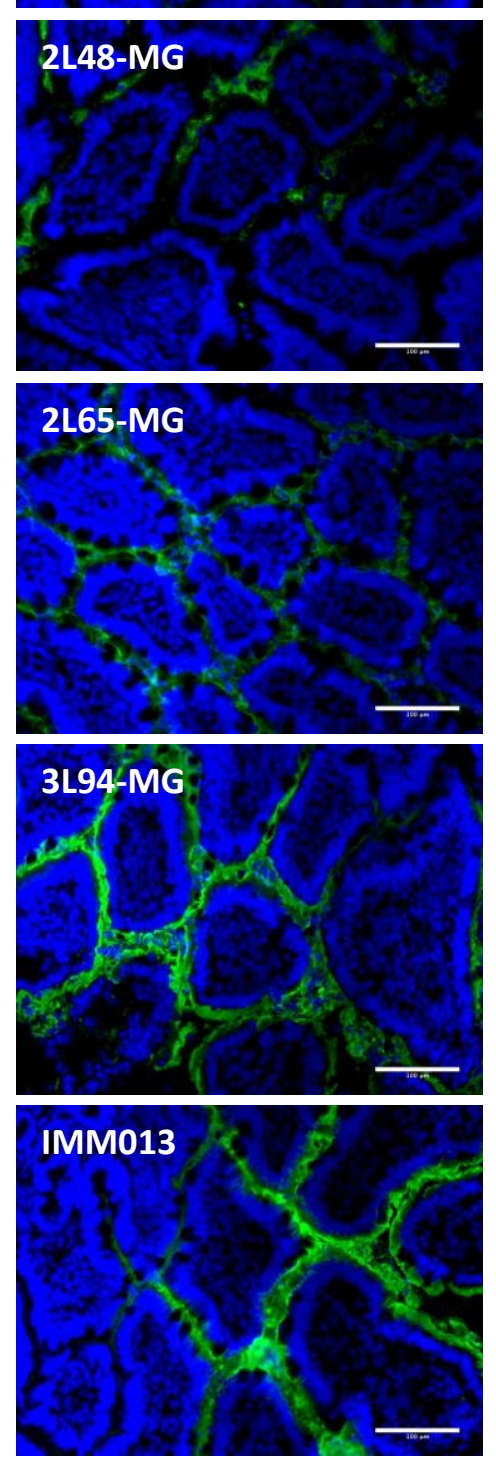
Supplementary Figure S4. Sections from jejunal loops of three pigs. Merged images stained with goat anti-mouse (FITC, green) and counterstained with Hoechst (blue). Scale bars: $100 \mu \mathrm{m}$. 
Pig 1
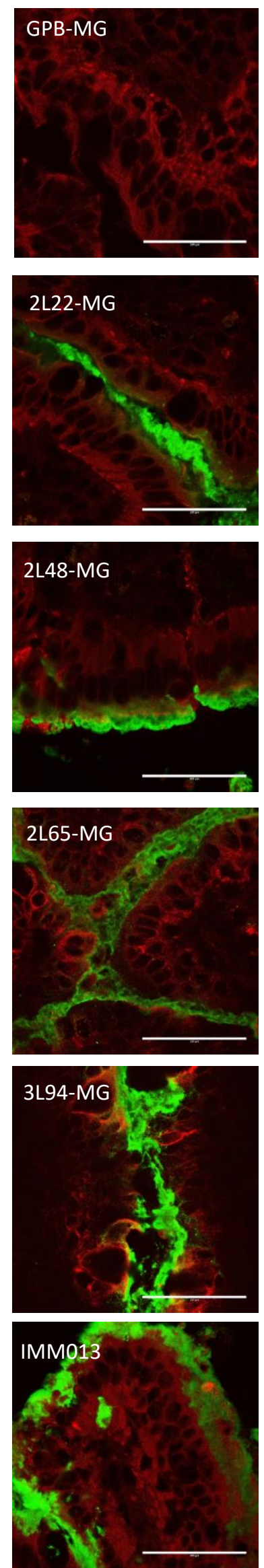

Pig 2
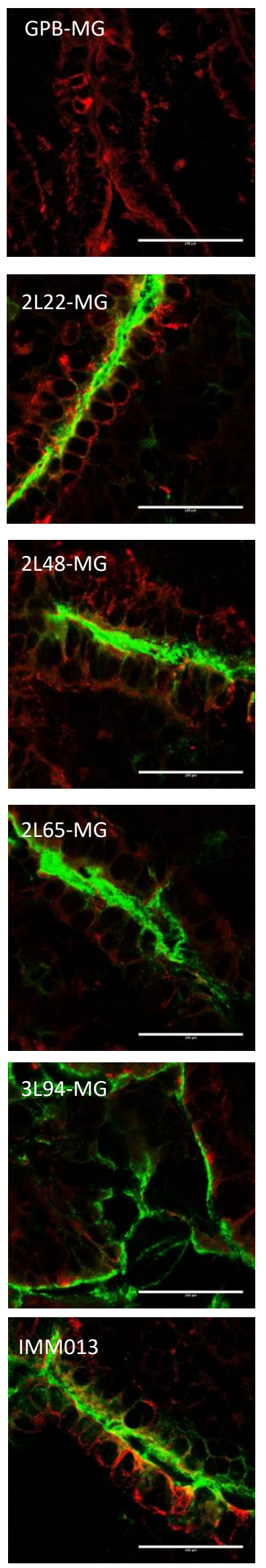

Pig 3
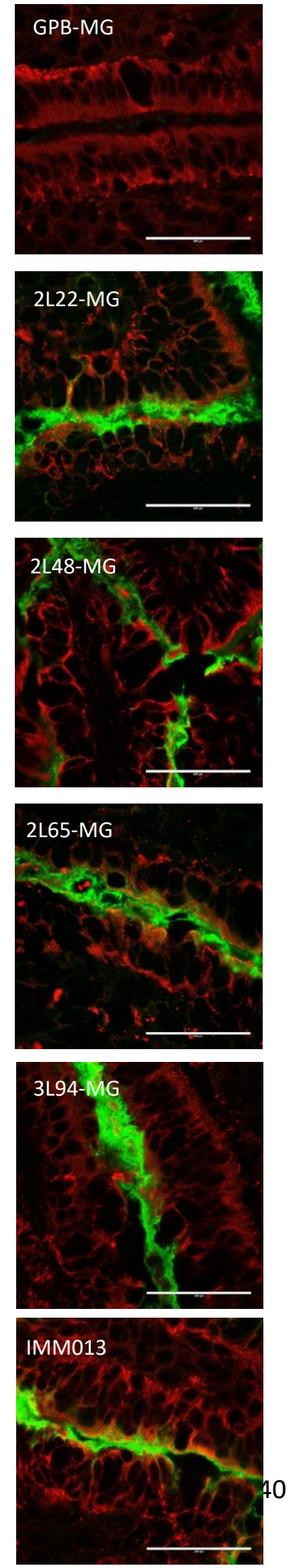
Supplementary Figure S5. Sections from jejunal loops of three pigs. Merged confocal images stained with goat anti-mouse (FITC, green) and counterstained with anti-cytokeratin TexasRed (red). Scale bars: $100 \mu \mathrm{m}$. 

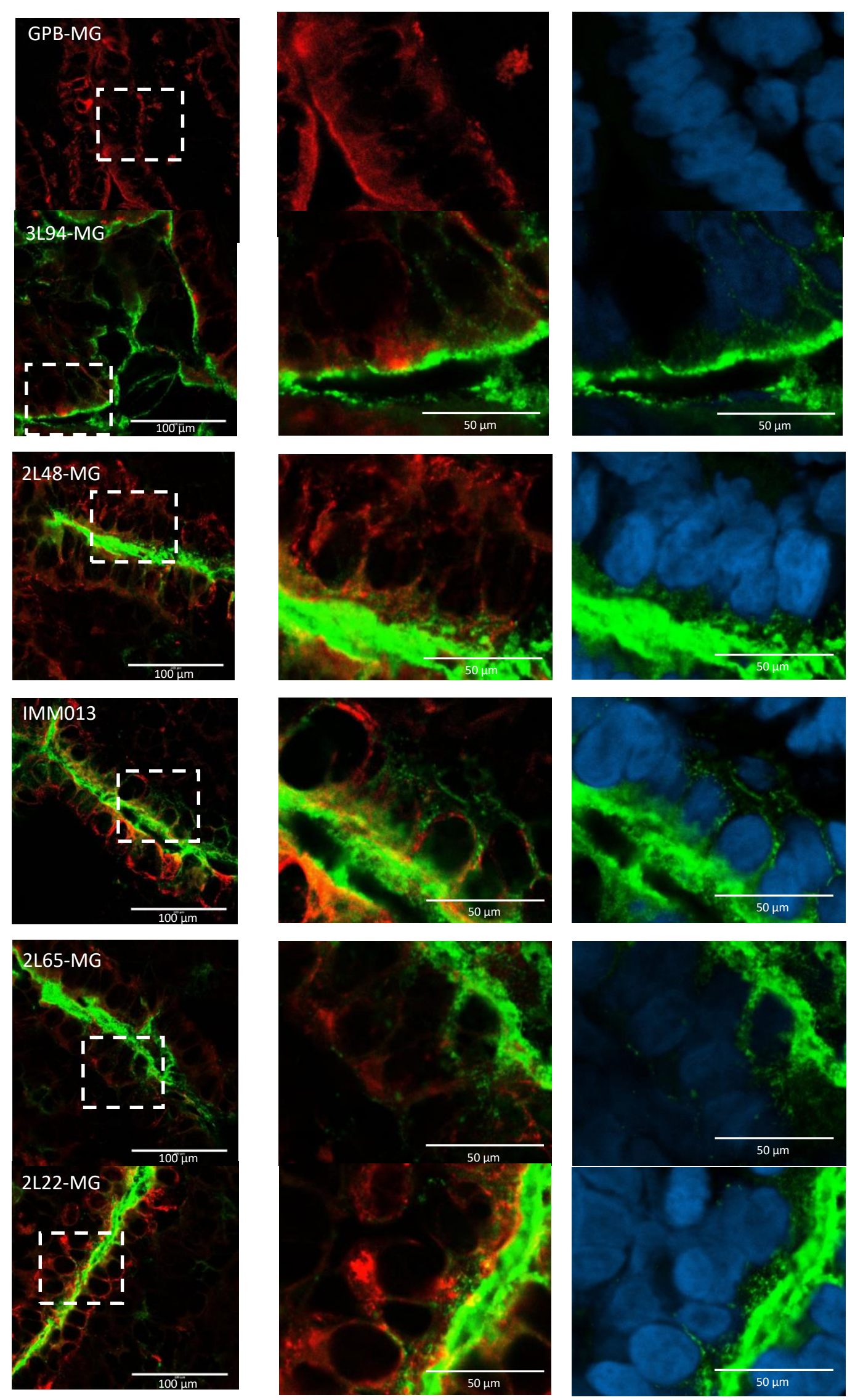
Supplementary Figure S6. Gut ligated loop experiment to determine the uptake of VHH-MG by enterocytes. Sections from jejunal loops stained with sheep anti-mouse IgG-FITC (green) and counterstained with anti-cytokeratin-TexasRed (red) or hoechst (blue). Confocal images with representative zooms (square boxes) show the uptake of VHH-MGs and IMM013 by small intestinal enterocytes. Representative of three independent experiments. (scale bars: $100 \mu \mathrm{m}$; scale bars for zoom: $50 \mu \mathrm{m}$ ). 